\title{
Non-spherical osmotic motor: chemical sailing
}

\author{
Sergey Shklyaev ${ }^{123}$, John F. Brady and Ubaldo M. Córdova-Figueroa \\ 'Department of Chemical Engineering, University of Puerto Rico - Mayagüez, Mayagüez, PR 00681, \\ USA \\ ${ }^{2}$ Divisions of Chemistry \& Chemical Engineering and Engineering \& Applied Science, California \\ Institute of Technology, Pasadena, CA 91125, USA \\ ${ }^{3}$ Institute of Continuous Media Mechanics, Ural Branch of the Russian Academy of Sciences, Perm, \\ 614013, Russia
}

(Received 1 October 2013; revised 28 March 2014; accepted 31 March 2014;

first published online 1 May 2014)

The behaviour of a non-spherical osmotic motor - an axisymmetric catalytic particle self-propelling in a dilute dispersion of reactant particles - is considered. In contrast to a conventional osmotic motor that creates differences in concentration, and hence in osmotic pressure, due to asymmetry in reaction rate along its surface (e.g. a Janus particle with reactive and non-reactive patches), a non-spherical particle is able to move even with uniform chemical activity on its surface. For small departures from a sphere the velocity of self-propulsion is proportional to the square of the non-sphericity or distortion of the particle shape. It is shown that the inclusion of hydrodynamic interactions (HI) may drastically change the self-propulsion. Except for very slow chemical reactions, even the direction of self-propulsion changes with and without HI. Numerical calculations at finite non-sphericity suggest that the maximum velocity of self-propulsion is obtained by a sail-like motor shape, leading to the name 'chemical sailing'. Moreover, no saturation in the speed of propulsion is found; the motor velocity increases as the area of this 'sail' grows and its thickness decreases. The self-propulsion of a non-spherical particle releasing products of a chemical reaction - a constant flux motor - is also considered.

Key words: colloids, low-Reynolds-number flows, self-propulsion

\section{Introduction}

The self-propulsion of small micron-scale particles is an area of great current interest, with applications in biology, chemistry, medicine and the design of smart materials. Catalytic nanomotors are self-propelling particles that operate by converting chemical energy into translational motion in a viscous medium via a surface chemical reaction (Sengupta, Ibele \& Sen 2012). In most studies, either experimental (Paxton et al. 2006; Howse et al. 2007) or theoretical (Golestanian, Liverpool \& Ajdari 2005, 2007; Córdova-Figueroa \& Brady 2008; Brady 2011; Córdova-Figueroa, Brady \& Shklyaev 2013), net propulsion is obtained by a non-uniform distribution of chemical reactivity on the motor surface (e.g. a half-reactive spherical Janus particle). The variation of the reactivity breaks symmetry and creates a non-uniform concentration

† Email address for correspondence: jfbrady@ caltech, edu 
distribution of reactants and products (solute) in the solution adjacent to the motor, which can result in an imbalanced interactive force between the motor and solute, leading to self-propulsion. From a continuum perspective (Golestanian et al. 2005, 2007) this imbalance gives rise to self-diffusiophoresis (Anderson 1989), while from a more detailed fined-grained colloidal perspective (Córdova-Figueroa \& Brady 2008; Brady 2011; Córdova-Figueroa et al. 2013) the imbalance results in a net osmotic pressure exerted on the motor due to the non-equilibrium distribution of solute. The conditions under which the two approaches agree are discussed in detail by Brady (2011).

A system geometrically (or topologically) different from but similar to the Janus particles discussed above is formed by a pair of firmly connected, but different, particles (e.g. catalytic and non-catalytic ones). Such a system was studied by Rückner \& Kapral (2007) and Thakura \& Kapral (2010) via molecular dynamics and by Popescu, Tasinkevych \& Dietrich (2011) within the continuum perspective. As before, the main principle giving rise to motion is an object with a non-uniform reactivity along its surface which produces a local gradient of solute concentration.

Another way to achieve self-propulsion, however, is to break the symmetry in the solute distribution via the shape of the particle rather than its reactivity. This was first appreciated by Wei \& Jan (2010) who, following the approach of Córdova-Figueroa \& Brady (2008), showed that a non-spherical particle with uniform reactivity could move autonomously. This perspective may have far reaching consequences, for one can use motors with uniform chemical reactivity that are easier to manufacture and still obtain self-propulsion. Motion due to shape asymmetry may also be important in biological systems; rarely is a molecular complex spherical. Unfortunately, the analysis by Wei \& Jan (2010) has some technical errors: they studied the motion for small non-sphericity and missed a term, which mistakenly led to self-propulsion for a translation of the non-deformed spherical motor, with the self-propulsion velocity proportional to this translation. As shown below, self-propulsion occurs at second order in the deformation from a spherical particle.

The purpose of this paper is to provide a complete derivation and analysis of 'shape-propulsion'. The problem is approached from the colloidal perspective by first extending the analysis of Brady (2011) to non-spherical particles. As shown by Brady (2011) for spherical particles in the limit when the solutes (reactants and/or products) are much smaller than the motor, the inclusion of the hydrodynamics only rescales the velocity of self-propulsion calculated by Córdova-Figueroa \& Brady (2008), who neglected hydrodynamic interactions (HI). In contrast, for a non-spherical motor the difference between the two limiting cases, (i) no $\mathrm{HI}$ and (ii) full HI, is not simply a rescaling of the motor speed, but may be crucial - even the direction of self-propulsion can change! In this paper we consider both small departures from sphericity, where results can be obtained analytically, and large departures from sphericity, which require a numerical approach for both no $\mathrm{HI}$ and full $\mathrm{HI}$.

The results of the calculations suggest that the optimum shape that gives the largest velocity for a given motor volume is a thin - to maximize the surface area for reaction - highly curved - to isolate one side of the surface from the reactants - shape, much like a sail; hence the term 'chemical sailing'. The motor 'sails' along in the 'wind' created by the non-uniform solute concentration due to the uniform chemical reaction on the motor surface.

In the analysis below we follow the work of Córdova-Figueroa \& Brady (2008) for a first-order irreversible chemical reaction and show that it can be extended to more general reactions where both product and reactant species are present in the solution. 
In particular, such a reformulation applies to a motor releasing product particles at its surface - a constant flux motor. Wei \& Jan (2010) also analysed this case, but used a negative reaction rate to model the emission of product particles, which is potentially unstable; the higher concentration of products intensifies the reaction and further magnifies the concentration.

We also show in appendix A how the classical 'continuum' approach can be derived (and thus justified) from the colloidal perspective when surface chemical reactions are present, something that was implicitly assumed in prior studies based on the continuum approach. This colloidal versus continuum perspective was discussed in detail by Brady (2011) in the context of classical diffusiophoresis (Anderson 1989) and is here extended to reactive particles of arbitrary shape.

The paper is organized as follows. The problem is developed in $\S 2$ within the colloidal description, where the approach of Brady (2011) is extended to non-spherical motors (and solute) and a general formula applicable for arbitrary shape, reactivity, interactive potential, etc. is given (see (2.3)). In $\S 3$ the limit of small non-sphericity is studied analytically, and numerical solutions for finite departures from a sphere are taken up in $\$ 4$, all for the case of an irreversible first-order chemical reaction occurring uniformly on the motor surface. It is shown that the motor speed has the general form expected for self-diffusiophoresis: $U=(2 / 9)\left((a+\delta)^{2} k_{B} T / \eta\right)\left(n^{\infty} / a\right) \times$ $V$ (shape, $\Delta, D a$ ), where $a$ is the motor size (e.g. the radius of the sphere with the same volume as the non-spherical motor; see $\S_{2}$ ), $\delta$ is the interactive length between the solute and the motor surface, $k_{B} T$ is the thermal energy, $\eta$ is the viscosity of the fluid and $n^{\infty}$ is the concentration of the reactant far from the motor $\left(n^{\infty} / a\right.$ being the scale of the concentration gradient). The non-dimensional 'motor speed' $V$ is a function of the motor 'shape', the ratio $\Delta=\delta / a$, and the Damköhler number $D a=$ $\kappa(a+\delta) / D_{b}$ comparing the rate of reaction to diffusion of the reactants; here, $\kappa$ is the first order rate constant with units of length/time, and $D_{b}$ is the diffusivity of the reactants. The parameter $\Delta$ controls the extent to which $\mathrm{HI}$ between the reactant and the motor influence the motor's motion: for no HI, $\Delta \gg 1$ and $V$ is independent of $\Delta$. For full HI, $\Delta \ll 1$ and $V \sim \Delta^{2}$, giving $U \sim\left(\delta^{2} k_{B} T / \eta\right)\left(n^{\infty} / a\right)$, as is customary for diffusiophoresis in the thin interfacial limit (Brady 2011). We consider a variety of motor shapes, ranging from elongated to flattened, and with different extents of convex and concave surface regions (see figure 2). Figure 9 gives a general sense of the direction and magnitude of the velocity of self-propulsion both with and without $\mathrm{HI}$ for representative asymmetric motor shapes.

In $\S 5$ we consider a motor that releases product particles from its surface, a so-called constant flux motor. The non-uniformly reactive spherical constant flux motor was recently discussed by Córdova-Figueroa et al. (2013) from the colloidal perspective. We show that the non-spherical constant flux motor can be recovered as a special limit of an irreversible chemical reaction and the motor speed is shown to take the form $U_{f}=(2 / 9)\left((a+\delta)^{3} k_{B} T /(a \eta)\right)\left(j_{s} / D_{P}\right) \times V_{f}($ shape, $\Delta)$, where $j_{s}$ is the fixed flux of products from the motor surface and $D_{P}$ the diffusivity of the products. There is no Damköhler number and so the non-dimensional 'motor speed' $V_{f}$ depends only on the shape and $\mathrm{HI}$ through $\Delta$. Interestingly, in contrast to the reactive motor, where HI affect not only the magnitude of the motor speed but also its direction, for a fixed flux motor generally only the magnitude of the speed is affected by HI, with $V_{f} \sim \Delta^{2}$, as for a reactive motor for small $\Delta$. The same class of motor shapes is considered for the constant flux motors, and the direction and magnitude of motor velocities with and without $\mathrm{HI}$ are shown in figure 12 .

Finally, we close in $\S 6$ by showing that the colloidal perspective leads naturally to various types of motor motion that all follow from the same basic physics and 


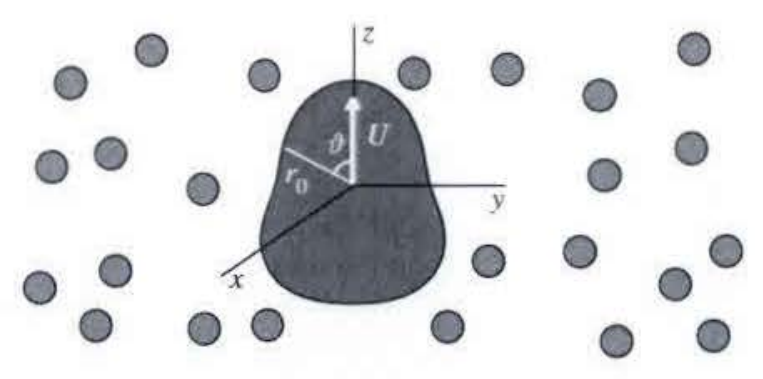

FIGURE 1. (Colour online) Definition sketch for non-spherical motor.

description and that can be described in an appealing figurative manner, namely: chemical swimming, sailing, surfing, squirming and swarming.

\section{Problem formulation}

We adopt the colloidal approach and model both the motor and the reactive solute species as colloidal particles dispersed in a continuous solvent so that the well-established equations of colloidal dynamics apply (Russel, Saville \& Schowalter 1989). As shown by Brady (2011), when two spherical colloidal particles exert a mutual interactive force on one another, $\boldsymbol{F}_{1}=-\boldsymbol{F}_{2}=\boldsymbol{F}_{12}(\boldsymbol{r})$, this causes the particles to move. Whether this interactive force is due to an actual colloidal interparticle potential, $\boldsymbol{F}_{12}^{(h)}=-\nabla_{1} \Phi$, or due to the relative Brownian motion of the two particles, $\boldsymbol{F}_{12}^{(B)}=-k_{B} T \partial \ln P\left(x_{1}, \boldsymbol{x}_{2}\right) / \partial \boldsymbol{x}_{1}=k_{B} T \nabla_{r} \ln g$, where $P\left(\boldsymbol{x}_{1}, \boldsymbol{x}_{2}\right)=n_{1} n_{2} g(\boldsymbol{r})$ is the joint probability density of their centres, does not matter. Here, $n_{1}$ and $n_{2}$ are the (uniform) number density of particles 1 and 2 , and $g(\boldsymbol{r})$ is the pair-distribution function. At the Smoluchowski level of colloidal dynamics the statistical or 'thermodynamic' Brownian force appears as the gradient of the probability density. Here, the motor particle is denoted particle ' 1 ', the reactive solute is denoted as ' 2 ', and $\boldsymbol{r}=\boldsymbol{x}_{2}-\boldsymbol{x}_{1}$ is the coordinate of a solute in a reference frame attached to the motor (see figure 1). The velocity of the motor particle when averaged over the probability distribution of the reactive solute particles is given by

$$
\boldsymbol{U}=n^{\infty} k_{B} T \int \boldsymbol{M} \cdot \nabla(\hat{\Phi}+\ln g) g \mathrm{~d} V .
$$

In (2.1), $n^{\infty}$ is the uniform number density of reactive solute far from the motor, $\hat{\Phi}$ is the interparticle potential normalized by the thermal energy $k_{B} T$ and $\boldsymbol{M}=\boldsymbol{M}_{11}-\boldsymbol{M}_{12}$ is the hydrodynamic mobility giving the velocity of particle 1 (the motor) due to a force acting on it, $\boldsymbol{M}_{11}$, and due to a force acting on particle 2 (the solute), $\boldsymbol{M}_{12}$. The integration is over all space accessible to particle 2. If there is more than one solute species (e.g. product particles), then an equation analogous to (2.1) is needed for each species and the probability density $g$ must be interpreted as the joint probability density for solute ' 2 ' relative to motor ' 1 ' in the presence of a third species, etc. The only approximation of diluteness made is in using the two-particle hydrodynamic mobilities in (2.1), and even this restriction can be relaxed (Brady 2011).

Although (2.1) was derived by Brady (2011) for spherical particles, the exact same formula applies for non-spherical particles, with the hydrodynamic mobilities now those corresponding to two interacting non-spherical particles and the integration to be interpreted as over the accessible volume in position and orientation space of 
(a)

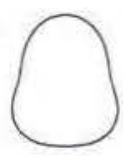

(b)

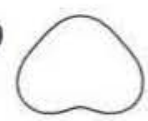

(c)
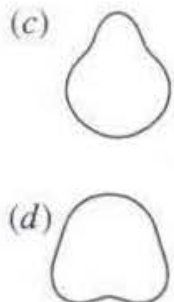

(e)

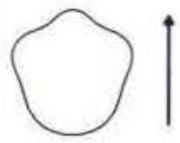

$(f) \bigcirc$

FIGURE 2. The shape of the slightly non-spherical particle for $n=2(a, b), n=3(c, d)$ and $n=4(e, f)$. For illustration purposes we show rather large deformations: $\left|\alpha_{n}\right|=\left|\alpha_{n+1}\right|=0.2$, with the other coefficients in (3.2) zero. For upper panels both $\alpha_{n}$ and $\alpha_{n+1}$ are positive; for $\Delta \gg 1$, the motor moves along the $z$ direction (see the arrow to the right). For lower panels the projection onto the even Legendre polynomial is negative; the particle moves opposite to the $z$ direction for $\Delta \gg 1$.

the solute relative to the motor (whose orientation is taken to be fixed, see below). Similarly, the gradient operator is in both position and orientation space and the probability density $g$ is for a given orientation and position of the solute relative to the motor. In this work we consider spherical reactive solute particles of radii $b$ but a non-spherical motor with characteristic size $a$ (being equal to the radius of the sphere of the same volume as the motor; see below for the precise definition) as illustrated in figure 1. Thus, the 'orientation' of the solute is not relevant; all that matters is the separation of the solute from the motor surface.

In the absence of a chemical reaction at the motor surface, the distribution of the reactant solute about the motor is given by the equilibrium Boltzmann distribution $g^{e q} \propto \mathrm{e}^{-\hat{\phi}}$, and the net interactive force $\boldsymbol{F}_{12}=-\nabla\left(\hat{\Phi}+\ln g^{e q}\right) \equiv 0$ is identically zero and there is no net motion of the motor, $\boldsymbol{U}=0$. This is, of course, as it should be whatever the motor shape there is no net force arising from the distribution of solute about it at equilibrium. (Note that the normalization constant for the pair-probability does not enter into the final expression for the motor velocity given below.)

When the reactive solute distribution is driven out of equilibrium by a surface chemical reaction there will, in general, be a non-zero interactive force and there may be net motion of the motor. Denoting the departure of the distribution function from equilibrium by

$$
g=g^{e q}(1+f)=\mathrm{e}^{-\hat{\phi}}(1+f),
$$

the net motor velocity becomes

$$
\boldsymbol{U}=n^{\infty} k_{B} T \int \mathrm{e}^{-\hat{\phi}} \boldsymbol{M} \cdot \nabla f \mathrm{~d} V .
$$

The local osmotic pressure of the reactive solute is defined as

$$
\Pi(\boldsymbol{r}) \equiv n(\boldsymbol{r}) k_{B} T=n^{\infty} k_{B} T g(\boldsymbol{r}),
$$

and thus (2.3) can also be written as

$$
\boldsymbol{U}=\int \boldsymbol{M} \cdot \mathrm{e}^{-\dot{\phi}} \nabla\left(\Pi \mathrm{e}^{\hat{\phi}}\right) \mathrm{d} \boldsymbol{V},
$$

showing that the motor motion is caused by gradients in the local osmotic pressure of the reactant (weighted by the reactant-motor interparticle potential) times the local relative mobility of the reactant-motor pair. 
For convenience in the analysis we now restrict consideration to reactive solutes that are much smaller than the motor, $\lambda \equiv b / a \ll 1$, and take the simplest form of interaction between the motor and the solute, namely, the solute particle is excluded from approaching the motor surface closer than a distance $\delta$ by a hard-particle repulsive potential. The chemical reaction also occurs at this separation distance $\delta$. This choice avoids the need to know anything precise about the solute-motor interactions - a single parameter, $\delta$, characterizes them, rather than both a length and an amplitude, as would be necessary with a soft potential. Integration of (2.5) (or (2.3)) by parts gives

$$
\boldsymbol{U}=-\oint_{S_{\epsilon}} \boldsymbol{M} \cdot \boldsymbol{n} \Pi \mathrm{d} S,
$$

where the integration is over the contact surface $S_{c}$ at a distance $\delta$ from the actual (the no-slip hydrodynamic) surface $S_{h}$ of the motor, and $\boldsymbol{n}$ is the external normal vector to the motor surface. In writing (2.6) we have neglected the volume integral proportional to $\boldsymbol{\nabla} \cdot \boldsymbol{M}$ because it is $\boldsymbol{O}\left(\lambda^{3}\right)$ even for non-spherical particles and is small (and note that there is no potential because $\hat{\Phi}=0$ for hard-particle interactions outside and at $S_{c}$ ).

From the well-known symmetry properties of the hydrodynamic mobility functions $\boldsymbol{M}_{12}=\boldsymbol{M}_{21}$, where the latter is the velocity of the solute particle due to a force on the motor, which, for small solute particles, is just the fluid velocity under a unit force imposed on the motor (correct to $O\left(\lambda^{3}\right)$ ). Thus, $\boldsymbol{M}=\boldsymbol{M}_{11}-\boldsymbol{M}_{21}$ gives the local fluid velocity relative to a motor particle subject to a unit force: $\boldsymbol{u}=-\boldsymbol{M} \cdot \boldsymbol{F}$. This permits another interpretation of the motor velocity: the osmotic pressure times the normal is a local force density per unit area on the motor and this force density multiplied by $\boldsymbol{M}$ is the local fluid velocity relative to the motor, often denoted as a 'slip' velocity, i.e. $-\boldsymbol{M} \cdot(\boldsymbol{n} \Pi)=-\boldsymbol{u}^{\text {slip }} /$ Area, where 'Area' is the local motor surface area element. The net velocity of the motor is thus the slip velocity averaged over the motor surface. This result should be familiar from the phoretic motion of spherical particles in the thin interfacial limit (Anderson 1989), where the reciprocal theorem for Stokes flow gives $\boldsymbol{U}^{\text {phoretic }}=-\oint \boldsymbol{u}^{\text {slip }} \mathrm{d} S /\left(4 \pi a^{2}\right)$, in agreement with (2.6) for a spherical motor. Note that this fluid velocity is evaluated at the contact surface $S_{c}$, which is everywhere a distance $\delta$ from the hydrodynamic no-slip surface $S_{h}$. To relax this condition, i.e. for $\delta \rightarrow 0$, see Brady (2011). Note that this notion of an integral of the slip velocity is also applicable to (2.5), where now the force density, $\mathrm{e}^{-\hat{\phi}} \nabla\left(\Pi \mathrm{e}^{\hat{\phi}}\right)$, is distributed throughout the volume surrounding the motor.

We shall take a simple form for the chemical activity of the motor: the reactive solute undergoes a first-order irreversible chemical reaction uniformly on the motor surface: $\boldsymbol{n} \cdot \boldsymbol{j}=-\kappa n=-\kappa n^{\infty} g$ on $S_{c}$, where $\boldsymbol{j}$ is the solute flux relative to the motor. Thus, for dilute small reactant particles $(\lambda \ll 1)$ the steady concentration distribution of the reactants satisfies the diffusion equation

$$
\begin{aligned}
\nabla^{2} g & =0, \\
\boldsymbol{n} \cdot \nabla g & =\text { Dag on } S_{c}, \\
g & \rightarrow 1 \quad \text { as } r \rightarrow \infty .
\end{aligned}
$$

where all lengths have been scaled with the characteristic size $r_{c}$, introduced such that the excluded volume of the motor is equal to $(4 \pi / 3) r_{c}^{3}$. The three typical lengths, $a, r_{c}$ and $\delta$ are related via the equality $r_{c}=a+\delta=a(1+\Delta)$. The non-dimensional reaction rate is given by the Damköhler number $D a=\kappa r_{c} / D_{b}$, where $D_{b}=k_{B} T /(6 \pi \eta b)$ 
is the diffusivity of a reactant particle. We have also assumed that the Péclet number for the solute $P e=U r_{c} / D_{b}$ is small, where $U$ is the yet-to-be-determined speed of the motor, and the effect of convective motion of the fluid surrounding the motor on the solute transport can be neglected. Typical motor speeds for micron-sized catalytic motors are a few microns per second (Paxton et al. 2006; Howse et al. 2007), giving Péclet numbers of $O\left(10^{-3}\right)$ for nanoscale solutes. The effects of convection can be included, as was done by Córdova-Figueroa \& Brady (2008) in their study of osmotic propulsion due to an asymmetric chemical reaction, but will be left for a future study.

The time to reach a steady-state concentration profile, $\tau_{g} \sim a^{2} / D_{b}$, is short compared to the time it takes the motor to change its orientation due to Brownian rotation, $\tau_{r} \sim 1 / D_{r} \sim \eta a^{3} /\left(k_{B} T\right)$, i.e. $\tau_{g} / \tau_{r} \sim b / a=\lambda \ll 1$, so that a net translation of the motor is possible. At very long times $\left(t \gg \tau_{r}\right)$ the motor will undergo a random walk as it reorientates due to Brownian rotation and its long-time motion will be diffusive with an effective translational diffusivity given by $D_{t}^{e f f}=D_{a}+U^{2} /\left(6 D_{r}\right)$ (Golestanian et al. 2007; Howse et al. 2007), where $D_{a}$ is the average (over all orientations) translational Brownian diffusivity of the motor, $U$ is the steady reaction-induced speed of the motor and $D_{r}$ is the (averaged) rotational diffusivity of the motor.

The appropriate length scale for the motor is $r_{c}$, rather than $a$, which defines the actual motor surface $S_{h}$ where the hydrodynamic no-slip boundary condition applies. Thus, when we refer to the characteristic motor size as the radius of the sphere with the equivalent volume we will be taking $r_{c}$ (not $a$ ) as the fundamental length. For small interactive lengths, $\delta \ll a$, this distinction is not important; the opposite situation, $\delta \gg a$, requires more care and is discussed below.

A hard-particle interaction potential at $S_{c}$ means that the reactive solute cannot get any closer to the motor surface than $r_{c}(>a)$ and therefore the chemical reaction must also occur at $S_{c}$ rather than at the hydrodynamic surface at $S_{h}$. Clearly, a truly infinite hard-particle repulsive potential would prevent any reaction from occurring at the motor surface, for the reactant could never overcome the barrier and reach the surface. (This is explained further in appendix A from the continuum perspective.) However, an actual chemical reaction occurs on a molecular scale and details at this level are beneath the colloidal and/or continuum descriptions. In contrast, the flux of reactant to the motor surface and the non-uniform reactant concentration field this induces occur on the colloidal scale and are the origin of the motor's motion. The hard-particle potential is used for convenience so that only the interactive length $\delta$ is needed rather than both an amplitude and range if a soft potential were used.

Finally, although we have assumed a simple irreversible first-order reaction, Córdova-Figueroa \& Brady (2008) showed that for more general chemistries where reactants produce ' $s$ ' product particles upon reaction the only modification is that (2.6) for the motor velocity must be multiplied by the stoichiometry/diffusivity factor $\left(1-s D_{R} / D_{P}\right)$, where $s$ is the stoichiometry of the reaction and $D_{R} / D_{P}$ is the ratio of the reactant to product diffusivities. (It is straightforward to show that the same relation holds with $\mathrm{HI}$.) In particular, the important limiting case of a motor that releases product particles corresponds to $D_{R} \gg D_{P}$ : the reactant particles diffuse so fast that their distribution is nearly uniform, $g \approx 1$, and therefore the flux of products $j_{s}=s \kappa n^{\infty}$ is constant along the motor surface. This case can also be viewed as that corresponding to a very slow (for the reactant species) chemical reaction, or small $D a$, with $D_{R}$ in place of $D_{b}($ see $\S 5)$.

It should be noted that Wei \& Jan (2010) modelled the case of product emission as a negative reaction rate, limiting consideration to $-1<D a<0$. Although within this interval of Damköhler number a steady solution for the concentration distribution 
is possible for a sphere, for other geometries a negative $\kappa$ (and, hence, $D a$ ) leads to either a negative concentration or its unbounded growth in time, which are clearly unphysical. A negative $D a$ cannot be used in general and does not correspond to a constant flux motor.

The problem now is composed of two parts: (i) hydrodynamics around the non-spherical motor and (ii) the distribution of the reactant solute $g$ about the motor. Given a motor geometry, the no-slip surface $S_{h}$ and the dimensionless separation distance $\Delta=\delta / a$, the hydrodynamic mobilities $\boldsymbol{M}$ are completely determined from a solution of the low-Reynolds-number or Stokes equations. Although the solution of Stokes equations for a body of revolution analysed below is a classical problem (see, e.g. Payne \& Pell 1960), the calculation of $\boldsymbol{M}$ involves details usually omitted in the literature, and therefore additional calculations are needed. The departure of the reactant solute distribution from equilibrium due to the chemical reaction is a solution to the diffusion equation $(2,7)$ governed by the rate of reaction to diffusion, the Damköhler number, and the shape of the contact surface $S_{c}$.

For the sake of simplicity we consider an axisymmetric motor with the contact surface $S_{c}$. parameterized as

$$
r=r_{0}(\mu), \quad \mu=\cos \vartheta
$$

in spherical coordinates as shown in figure 1. For this parametrization the non-dimensional motor speed or Péclet number from (2.6) is given by

$$
\begin{aligned}
P e & =\frac{(1+\Delta)^{3}}{\lambda^{2}} \phi^{\infty} V . \\
V & =\frac{3}{2} \int_{-1}^{1} \tilde{M}_{z n} g\left(r_{0}, \mu\right) r_{0} \sqrt{r_{0}^{2}+\left(r_{0}^{\prime}\right)^{2}\left(1-\mu^{2}\right)} \mathrm{d} \mu .
\end{aligned}
$$

Here, $\phi^{\infty}=n^{\infty} 4 \pi b^{3} / 3$ is the volume fraction of the solute, $\tilde{M}_{z n}=(6 \pi \eta a) \boldsymbol{e}_{z} \cdot \boldsymbol{M} \cdot \boldsymbol{n}$ is the normalized component of the mobility and the prime denotes the derivative with respect to $\mu$. The normal vector is given by

$$
\boldsymbol{n}=\frac{r_{0} \hat{\boldsymbol{r}}-r_{0}^{\prime}\left(\boldsymbol{e}_{z}-\mu \hat{\boldsymbol{r}}\right)}{\sqrt{r_{0}^{2}+\left(r_{0}^{\prime}\right)^{2}\left(1-\mu^{2}\right)}}, \quad \hat{\boldsymbol{r}}=\frac{\boldsymbol{r}}{r} .
$$

All calculations are performed either under conditions of no $\mathrm{HI}(\Delta=\delta / a \rightarrow \infty)$ or full $\mathrm{HI}(\Delta=\delta / a \rightarrow 0)$. These limits are the only cases when the representation of both surfaces, $S_{c}$ and $S_{h}$ (given by $r=r_{0}^{(-)}(\mu)$ ), are simply connected one with the other. In the former case, $\Delta \gg 1, a$ is small in comparison with both $r_{c}$ and $\delta$. This means that $\delta \approx r_{0}(\mu)$ varies along the motor surface, whereas $S_{h}$ is formally given by $r_{0}^{(-)}=O(1 / \Delta) \ll 1$ ( $\Delta$ should be thought as the mean value of $\delta / a$ along the surface), so that the hydrodynamic core has a small size for the chosen length scale $r_{c}$. When $\mathrm{HI}$ are neglected the mobility is a constant for the given motor shape:

$$
\boldsymbol{e}_{z} \cdot \boldsymbol{M}=\boldsymbol{e}_{z} M_{z z}^{\infty}=\boldsymbol{e}_{z} \frac{\tilde{M}_{z z}^{\infty}}{6 \pi \eta a}
$$

and therefore

$$
\tilde{M}_{z n}=\tilde{M}_{z z}^{\infty} \boldsymbol{e}_{z} \cdot \boldsymbol{n},
$$


where $M_{z z}^{\infty}$ is the mobility of the body of revolution moving along its axis, which is the same as the velocity of the body when a unit force is applied to it. In calculations of $M_{z z}^{\infty}$ we set $r_{0}^{(-)}=r_{0}(\mu) / \Delta$.

In the other case of full HI, $\Delta=\delta / a \ll 1, r_{0}^{(-)}=r_{0}-\Delta /(1+\Delta) \approx r_{0}-\Delta+\Delta^{2}$ and $|\boldsymbol{M}| \sim \Delta^{2}$. (It is worth noting that this simple parametrization again leads to variation of $\delta$ along the motor surface. However, for small non-sphericity the difference between $r_{0}^{(-)}(\mu)$ and that corresponding to a constant separation $\Delta /(1+\Delta)$ between $S_{h}$ and $S_{c}$ is unimportant, whereas for finite non-sphericity $S_{h}$ is not needed, as will be explained below.) Recall that this case can be mapped to the continuum perspective for self-diffusiophoresis, because $\delta$ is proportional to the Derjaguin length $\lambda_{D}$, which is assumed small in the continuum approach (see appendix A).

It may seem strange that the Péclet number is proportional to the volume fraction of the solute $\phi^{\infty}$, which can be exceedingly small as it is proportional to the solute size cubed. However, the dimensional velocity of the motor $U$ is actually independent of the solute size $b$, as expected. The relevant factor in $(2.11)$ is $\phi^{\infty}(1+\Delta)^{3} / \lambda^{2}=$ $4 \pi n^{\infty} r_{c}^{3} b /(3 a)$, and thus the dimensional velocity is

$$
U=\frac{D_{b}}{r_{c}} \frac{4}{3} \pi n^{\infty} \frac{r_{c}^{3} b}{a} \times V=\frac{2}{9} \frac{r_{c}^{2} k_{B} T}{\eta}\left(\frac{n^{\infty}}{a}\right) V,
$$

which is independent of $b$. Indeed, $(2,16)$ has the form expected for phoretic motion, namely, $U \sim(\text { length })^{2} \times\left(k_{B} T / \eta\right) \times \nabla n^{\infty}$, with $\nabla n^{\infty} \sim n^{\infty} / a$ and (length $)^{2} \sim r_{c}^{2} V$. For full HI, $\Delta \ll 1, r_{c} \approx a, V \sim \Delta^{2}$ and $r_{c}^{2} V \sim \delta^{2}$, giving the common 'continuum' scaling in the thin interfacial limit. For no HI, $\Delta \gg 1, r_{c} \approx \delta \gg a$ and $V$ is independent of $\Delta$, which gives the behaviour shown by Córdova-Figueroa \& Brady (2008).

In the sections that follow, the quantity $V$, referred to as the "velocity of self-propulsion', is calculated for different values of the parameters $\Delta$ and $D a$, and various geometries of the motor. One must remember to multiply by the appropriate scale factors as in $(2.16)$ to get the actual motor velocity $U$.

\section{Small non-sphericity}

\subsection{The microstructure}

We first consider a near-spherical catalytic particle whose axisymmetric shape is described by a series of Legendre polynomials $P_{n}(\mu)$ :

$$
r_{0}=1+\epsilon \zeta_{1}+\epsilon^{2} \zeta_{2}, \quad \epsilon \ll 1,
$$

where

$$
\begin{aligned}
& \zeta_{1}=\sum_{n=2}^{\infty} \alpha_{n} P_{n}(\mu), \\
& \zeta_{2}=\alpha_{0} P_{0}(\mu)+\alpha_{1} P_{1}(\mu) .
\end{aligned}
$$

As a reminder, the first few Legendre polynomials are:

$$
P_{0}(\mu)=1, \quad P_{1}(\mu)=\mu, \quad P_{2}(\mu)=\frac{1}{2}\left(3 \mu^{2}-1\right), \quad P_{3}(\mu)=\frac{1}{2}\left(5 \mu^{3}-3 \mu\right), \ldots,
$$

and physically have the following meaning: $P_{0}(\mu)$ and $P_{1}(\mu)$ correspond to a change in the volume and the centre of the motor, respectively; $P_{2}(\mu)$ corresponds to a motor 
deformation to a spheroid (prolate for $\alpha_{2}>0$ and oblate otherwise); and $P_{3}(\mu)$ breaks the fore-aft symmetry - non-zero $\alpha_{3}$ corresponds to a motor deformation to a cone-like body (with smoothed rim and vertex).

The formal small parameter $\epsilon$ is a measure of the small non-sphericity. The coefficients $\alpha_{n}(n \geqslant 2)$, which appear in (3.2), parameterize the distorted surface; they are arbitrary. In contrast, the coefficients $\alpha_{0}$ and $\alpha_{1}$ are slaved; they have to be evaluated for given $\alpha_{n}(n \geqslant 2)$ in order to fix the volume of the particle and the position of its centre to be the same as for the sphere $r=1$. With accuracy $O\left(\epsilon^{2}\right)$ one arrives at

$$
\alpha_{0}=-\sum_{n=2}^{\infty} \frac{\alpha_{n}^{2}}{2 n+1}, \quad \alpha_{1}=-3 \sum_{n=2}^{\infty} C_{n} \alpha_{n} \alpha_{n+1}, \quad C_{n}=\frac{3(n+1)}{(2 n+1)(2 n+3)} .
$$

In the $O(\epsilon)$ expansion (3.2), a zeroth Legendre polynomial would correspond to a change in the radius of a spherical motor, which does not break symmetry and cannot lead to any net motion, and therefore its coefficient is set to zero to preserve the scale for the motor as $r_{c}$. A first Legendre polynomial would correspond to a simple translation (without deformation) of the centre of the sphere, as can been seen from the following: the equation for a sphere of unit radius whose centre is shifted a small distance, $\epsilon$, along the $z$-axis is

$$
x^{2}+y^{2}+(z-\epsilon)^{2}=1
$$

from which

$$
x^{2}+y^{2}+z^{2}=1+2 \epsilon z+O\left(\epsilon^{2}\right)
$$

or

$$
r=1+\epsilon P_{1}(\mu)+O\left(\epsilon^{2}\right) .
$$

A shift in the centre of the spherical motor does not break symmetry and cannot lead to motion; thus the coefficient of the first Legendre polynomial at $O(\epsilon)$ may (should) also be set to zero.

To study the self-propulsion of a near-spherical motor, both the concentration and the velocity $V$ are also expanded in powers of $\epsilon$ :

$$
g=g_{0}+\epsilon g_{1}+\epsilon^{2} g_{2}+\cdots, \quad V=\epsilon V_{1}+\epsilon^{2} V_{2}+\cdots .
$$

At zeroth order the problem for the concentration field is straightforward:

$$
\begin{aligned}
\nabla^{2} g_{0} & =0, \\
\frac{\partial g_{0}}{\partial r} & =\text { Dag }_{0} \quad \text { at } r=1, \\
g_{0} & \rightarrow 1 \quad \text { as } r \rightarrow \infty,
\end{aligned}
$$

with solution

$$
g_{0}=1+\frac{A}{r}, \quad A=-\frac{D a}{1+D a} .
$$

giving a local spherically symmetric depletion of reactant near the motor.

The boundary-value problem at first order and its solution are given in appendix B. It is clear that $V_{1}$ vanishes even if the projection of the surface distortion onto the zeroth and first Legendre polynomials are retained at $O(\epsilon)$ (Wei \& Jan 2010). Indeed, 
any net motion that is linear in the small departure from sphericity must arise from $P_{1}(\mu)$, as only this term has the proper symmetry for motion. However, there are two contributions to the motion that must be considered. First, the leading order undisturbed concentration field, which is spherically symmetric and centred on the spherical motor at the origin (see $(3,13)$ ), will generate a net force and motion when integrated over the 'distorted' shape coming from $P_{1}$ - this distortion we recognize from (3.6) is another sphere whose centre is shifted slightly along the $z$-axis. Second, there is also a distorted concentration field that must be integrated over the surface of the original sphere at the origin. This distorted concentration field is again that due to a sphere, but now at the shifted origin, and when integrated over the original sphere the second contribution precisely cancels the first one. (In appendix B we present the mathematics corresponding to this physical explanation.) Wei \& Jan (2010) only considered the second contribution and therefore incorrectly concluded that there was net motion at $O(\epsilon)$.

Hence, there is no autonomous motion linear in the non-sphericity, $V_{1}=0$, and thus one must proceed to higher order in the expansion in powers of $\epsilon$ for both the concentration and the self-propulsion velocity $V$. The boundary-value problem at second order and the part of its solutions $g_{2}^{(1)}$ needed for the further calculations are presented in appendix B.

Finally, the area element $\mathrm{d} S$, which appears in (2.12), can be approximated using the relation

$$
\frac{\mathrm{d} S}{2 \pi \mathrm{d} \mu}=r_{0} \sqrt{r_{0}^{2}+\left(r_{0}^{\prime}\right)^{2}\left(1-\mu^{2}\right)} \approx r_{0}^{2} \approx 1+2 \epsilon \zeta_{1}+O\left(\epsilon^{2}\right)
$$

to guarantee the $O\left(\epsilon^{2}\right)$ accuracy of the velocity calculation. Indeed, the neglected $O\left(\epsilon^{2}\right)$ terms contribute to $V$ only when multiplied by the spherically symmetric $g_{0}(1)=1+A$. The integral corresponding to such a product must be zero due to the obvious physical requirement that it would correspond to self-propulsion in the absence of a chemical reaction. (In order to calculate $V_{3}$ in $\$ 3.2 .2$ one has to continue the series for $\mathrm{d} S /(2 \pi \mathrm{d} \mu)$ to second order; this cumbersome result is not presented here.)

\subsection{No HI, limit of large $\Delta$}

In the limiting case $\Delta \gg 1, \tilde{M}_{z n}$ is given by $(2.15)$; for small non-sphericity the required component of the renormalized mobility is close to unity (Sampson 1891; Happel \& Brenner 1965)

$$
\tilde{M}_{z z}^{\infty}=1+\frac{1}{5} \epsilon \alpha_{2}+O\left(\epsilon^{2}\right) .
$$

(Comparing this expression for the force with that given in the cited papers, one has to take into account the difference between the Gegenbauer and Legendre polynomials and fix the particle volume properly.) This equation can be readily extracted from the analysis presented in appendix $C$.

In fact, however, the $O(\epsilon)$ correction is not needed within the current section. The integral for $V_{2}$ is of order $\epsilon^{2}$ and therefore the $O(\epsilon)$ term in $\tilde{M}_{z z}^{\infty}$ produces $O\left(\epsilon^{3}\right)$ corrections. (Moreover, in contrast to the expansion of $\mathrm{d} S /(2 \pi \mathrm{d} \mu)$, the correction to $\tilde{M}_{z z}^{\infty}$ is not even needed to calculate $V_{3}$ in $\S 3.2 .2$ in the absence of HI.) 

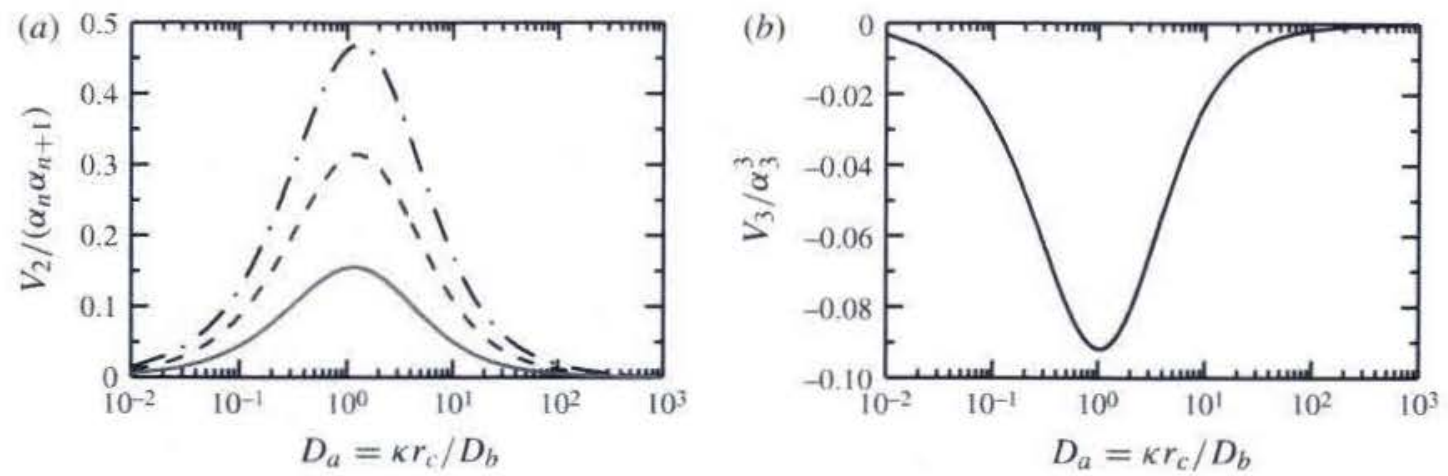

FIGURE 3. (Colour online) The velocity of self-propulsion for small non-sphericity $V_{j}=$ $P e\left(b / r_{c}\right)^{2} /\left(\phi^{\infty} \epsilon^{j}\right)(j=2,3)$ in the absence of HI. $(a)$ : the normalized velocity at second order in the perturbation theory $V_{2} /\left(\alpha_{n} \alpha_{n+1}\right),(3.16)$, depending on the Damköhler number $D a=\kappa r_{c} / D_{b}$ for $n=2,3$ and 4 (solid, dashed and dot-dashed lines, respectively). The particle shapes corresponding to these $n$ are shown in figure 2. (b): the normalized velocity at the third order of the perturbation theory $V_{3} / \alpha_{3}^{3},(3.22)$, versus the Damköhler number for the particular case $\alpha_{n}=0$ for $n=2$ and $n>3$.

\subsubsection{Quadratic effects}

Substituting $g_{0}, g_{1}, g_{2}$ and $\tilde{\boldsymbol{M}}$ into (2.12), one arrives at the following velocity of self-propulsion:

$$
\begin{aligned}
& V_{2}=\sum_{n=2}^{\infty} \alpha_{n} \alpha_{n+1} U_{n}, \\
& U_{n}=\frac{D a C_{n}}{1+D a}\left[\frac{n^{2}+2 n-2}{2+D a}-2+\frac{(3+D a)(n+2+2 D a)}{(n+1+D a)(n+2+D a)}\right],
\end{aligned}
$$

where the $C_{n}$ are defined in (3.5).

Since the velocity at second order is a superposition of pair combinations of neighbouring harmonics, one can consider the simplest case; when only a pair of coefficients $\alpha_{n}$ and $\alpha_{n+1}$ is non-zero in (3.2) for certain $n \geqslant 2$. The motor shapes for the lowest three values of $n: n=2,3,4$, are depicted in figure 2. In this figure the odd coefficients $\left(\alpha_{3}\right.$ and $\left.\alpha_{5}\right)$ are chosen positive; it is clear that changing their sign only reflects the motor with respect to the $x-y$ plane.

Equation (3.16) indicates that the particle changes the direction of its self-propulsion when one of the coefficients - either $\alpha_{n}$ or $\alpha_{n+1}$ - changes sign. As stated above, this effect is expected for the change of sign for the projection onto odd Legendre polynomials. The velocity reversal under change of sign for even Legendre polynomials, which indeed changes the particle shape (see the upper and lower rows in figure 2), is not universal; it fails with inclusion of cubic effects, as shown in $\S 3.2 .2$.

The resulting values of $U_{2}=V_{2} /\left(\alpha_{2} \alpha_{3}\right)$ are depicted in figure $3(a)$ for the motors shown in figure 2. As can be expected, the velocity of self-propulsion attains its maximum value at a certain value of $D a$, vanishing in the two limiting cases of slow and fast chemical reaction. Both of these limits are clear: in the absence of reaction $(D a=0)$ there is no reason to move at all, whereas for small $D a$ the difference in 
the osmotic pressure is proportional to the Damköhler number, which results in

$$
V_{2}(D a \ll 1)=\alpha_{n} \alpha_{n+1} D a \frac{3 n(n-1)(n+4)}{2(2 n+1)(2 n+3)} .
$$

At large $D a$ the concentration of the reactant particles (and thus the osmotic pressure) tends to zero near the motor surface and, again, the velocity of self-propulsion is small:

$$
V_{2}(D a \gg 1)=\frac{\alpha_{n} \alpha_{n+1}}{D a} \frac{3 n\left(n^{2}-1\right)}{(2 n+1)(2 n+3)} .
$$

The curves for different $n$ are qualitatively similar: the self-propulsion velocity becomes larger for larger $n$ (i.e. for more distorted, fanciful shapes). The maximum of $V_{2}$ is reached at $D a=D a_{m}$ close to unity, which grows slightly as $n$ increases ( $D a_{m}=1.155$ for $n=2, D a_{m}=1.268$ for $n=4$, and $D a_{m}=\sqrt{2} \approx 1.414$ for $n \gg 1$ ). The maximum values of $V_{2} /\left(\alpha_{n} \alpha_{n+1}\right)$ are $0.1552,0.3145,0.4686$ for $n=2,3,4$, respectively, i.e. for $n=3(n=4)$ the maximum velocity of self-propulsion is approximately two (three) times larger than for $n=2$. This tendency continues for all values of $n$; in particular for $n \gg 1(3.16)$ reduces to

$$
V_{2}(n \gg 1)=\alpha_{n} \alpha_{n+1} \frac{3 D a}{4(1+D a)(2+D a)} n .
$$

Thus, motors with small-scaled distortions (larger $n$ ) have higher self-propulsion velocities; their manufacture may pose a challenge, however.

\subsubsection{Cubic effects}

For the case when there is no pair of non-zero neighbouring harmonics in the shape representation (3.2) the effect is at least $O\left(\epsilon^{3}\right)$. The simplest distortion $\zeta$ that provides a self-propulsion velocity at that order includes one non-zero odd Legendre polynomial in (3.2). (It is clear that $\zeta_{1}$, which comprises only even Legendre polynomials, makes the motor symmetric with respect to the transformation $z \rightarrow-z$; no self-propulsion is possible then.) Let us consider as an example the case $\alpha_{3} \neq 0$ :

$$
r_{0}=1+\epsilon \alpha_{3} P_{3}(\mu)+\epsilon^{2} \alpha_{0}+\epsilon^{3} \alpha_{1} \mu,
$$

with $\alpha_{0}=-\alpha_{3}^{2} / 7$ given by (3.5) for $n=3$ and $\alpha_{1}=-36 \alpha_{3}^{3} / 385$.

The zero-, first- and second-order problems as well as the solutions for $g_{0}$ and $g_{1}$ are the same as presented in $\$ 3.2 .1$ and appendix B. Both the cumbersome solution for $g_{2}$ and the boundary-value problem governing $g_{3}$ are not presented here. The result of the calculation is

$$
V_{3}=-\frac{12 D a\left(41 D a^{2}+733 D a+2940\right) \alpha_{3}^{3}}{2695(1+D a)(2+D a)(4+D a)(5+D a)} .
$$

The corresponding velocity is shown in figure $3(b)$ - it is negative and again $\left|V_{3}\right|$ approaches a maximum at $D a$ close to unity $\left(\max \left|V_{3}\right|=0.09190 \alpha_{3}^{3}\right.$ at $\left.D a=1.034\right)$, vanishing for both slow

$$
V_{3}(D a \ll 1)=-\frac{18}{55} D_{a \alpha}^{3}
$$


and fast

$$
V_{3}(D a \gg 1)=-\frac{482 \alpha_{3}^{3}}{2695 D a}
$$

chemical reactions.

Equation (3.22) and the analysis in $\$ 3.2 .1$ allow one to combine both quadratic and cubic effects when $\alpha_{2} \neq 0$ and $\alpha_{3}>0$; see the shapes shown in figure $2(a)$ and 2(b). (Recall that a change of sign for $\alpha_{3}$ is unimportant.) In this case the velocity of self-propulsion is represented as a superposition of the contributions:

$$
V=\epsilon^{2} \alpha_{2} \alpha_{3} U_{2}+\epsilon^{3} V_{3} \text {. }
$$

where $U_{2}$ and $V_{3}$ are given by (3.17) and (3.22), respectively. Note that, formally, this equation is valid only for $\left|\alpha_{3}\right|^{4} \ll\left|\alpha_{2}\right| \ll\left|\alpha_{3}\right|$, because it contains neither a term proportional to $\alpha_{2}^{2} \alpha_{3}$ nor a term of the form $\alpha_{3}^{5}$. (It is clear that $\alpha_{3}^{4}$, as well as any other squared harmonic, does not contribute to the self-propulsion.)

Equation (3.25) predicts that an osmotic motor with the concave segment (see figure $\left.2(b)\left(\alpha_{2} \alpha_{3}<0\right)\right)$ moves with a higher velocity than that shown in figure $2(a)$ $\left(\alpha_{2} \alpha_{3}>0\right)$. Indeed, in the former case both the corrections are negative and the cubic term augments the self-propulsion found at second order. In contrast, in the latter case these two effects compete.

The fact that the motor shown in figure $2(b)$ moves faster has a simple explanation. The chemical reaction near the concave segment of the surface is more pronounced because of geometry - the volume adjacent to the catalytic surface is larger, and thus the concentration (and hence the osmotic pressure) is smaller there. In contrast, near the convex segments (at $\vartheta$ close to zero and to $2 \pi / 3$ ) the concentration and osmotic pressure are larger because the reaction zone is smaller there. The decrease in $\Pi$ near $\vartheta=\pi$ and its increase near $\vartheta=0$ push the particle downwards, whereas the increase in $\Pi$ near $\vartheta=2 \pi / 3$ only slightly hinders this motion. (The smallness of this contribution near $\vartheta=2 \pi / 3$ arises mainly from the smaller projection of the force $-\Pi n \mathrm{~d} S$ onto the $z$-axis, the direction of motion.)

\subsection{Full $\mathrm{HI}$}

Including $\mathrm{HI}$ into analysis one has to calculate the mobility component, $\tilde{M}_{z n}$; the concentration field $g\left(r_{0}\right)$ is unchanged. Details of this procedure are presented in appendix $C$. Using the recurrence formulas for the Legendre polynomials, the factor that enters the integrand in (2.12) needed to multiply $g\left(r_{0}\right)$ is

$$
\frac{\mathrm{d} S}{2 \pi \mathrm{d} \mu} \tilde{M}_{z n}\left(r_{0}\right) \approx-\mu L_{0}-\epsilon(n+1)\left(\frac{\alpha_{n} F_{n}}{2 n+1} P_{n+1}+\frac{\alpha_{n+1} F_{n+1}}{2 n+3} P_{n}\right) ;
$$

where at small $\Delta$

$$
F_{n}=\frac{3}{2} \Delta^{2} n(n+2), \quad F_{n+1}=-\frac{3}{2} \Delta^{2} n(n-1)
$$

and $L_{0}$ is given by (C.13).

It should be noted that, like the expansion of $\mathrm{d} S$ (see (3.14) and the discussion that followed), the solution is needed only with accuracy $O(\epsilon)$ to calculate the velocity of self-propulsion at $O\left(\epsilon^{2}\right)$. (Again, the $O\left(\epsilon^{2}\right)$ correction to $\tilde{M}_{z n} \mathrm{~d} S /(2 \pi \mathrm{d} \mu)$ appears in the integral (2.12) only with the factor $g_{0}(1)=1+A$, which is constant along the 


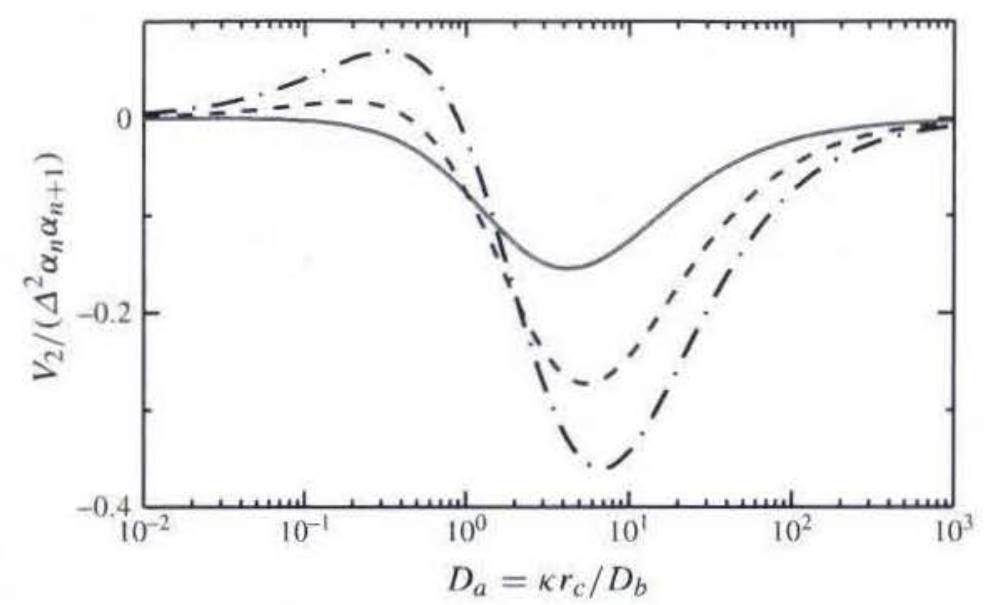

Figure 4. (Colour online) The rescaled velocity of self-propulsion for small nonsphericity, $V_{2}=P e\left(b / r_{c}\right)^{2} /\left(\phi^{\infty} \epsilon^{2}\right)$; for full HI, $\Delta \ll 1$. Variation of $V_{2} /\left(\Delta^{2} \alpha_{n} \alpha_{n+1}\right),(3,28)$, depending on the Damköhler number $D a=\kappa r_{c} / D_{b}$ for $n=2,3$ and 4 (solid, dashed and dot-dashed lines, respectively). The particle shapes corresponding to these $n$ are presented in figure 2 .

surface. A non-zero value of such an integral would correspond to a motor motion in a spatially uniform concentration field, which is not possible.)

Substituting the expansions for $g_{0,1,2}$ and $(3.26)$ into $(2.12)$, we obtain the following expression for $V_{2}$ :

$$
\begin{aligned}
& V_{2}=\Delta^{2} \sum_{n=2}^{\infty} \alpha_{n} \alpha_{n+1} \tilde{U}_{n} ; \\
& \tilde{U}_{n}=-\frac{3 C_{n} n(n-1) D a\left[3 D a^{2}+(n+7) D a+4-n^{2}\right]}{2(1+D a)(2+D a)(n+1+D a)(n+2+D a)} .
\end{aligned}
$$

First, as expected, in the limit of full HI the velocity of self-propulsion is proportional to $\Delta^{2}$, as it is for a non-uniformly reactive spherical Janus particle (see Brady 2011). Nevertheless, the structure of the resulting formula is similar to (3.16) in the absence of HI. The velocity is represented by a superposition of pair contributions of neighbouring Legendre polynomials, thus below only the effect of a separate pair of $n$th and $(n+1)$ th harmonics is discussed.

The variation of $V_{2} /\left(\alpha_{n} \alpha_{n+1} \Delta^{2}\right)$ is shown in figure 4 . As seen in the figure, the inclusion of $\mathrm{HI}$ profoundly changes the behaviour - even the direction of self-propulsion can be different! More precisely, for $n=2$ with HI the motor moves in the direction opposite to that without $\mathrm{HI}$ for any value of the Damköhler number. For $n>2$ and slow chemical reaction, $V_{2}$ has the same sign for $\Delta \rightarrow 0$ and for $\Delta \rightarrow \infty$, but at a certain value $D a=D a_{*}$ the velocity of self-propulsion with $\mathrm{HI}$ changes sign, whereas $V_{2}$ for no $\mathrm{HI}$ does not (see figure 3 ). The threshold value $D a_{*}$ increases as $n$ grows.

In order to explain the origin of this difference the particular case of the motor shown in figure $2(b)$ is considered. As discussed above, in the absence of $\mathrm{HI}$ the local (relative to $g_{0}$ ) decrease in concentration at $\vartheta=\pi$ and the relative increase at $\vartheta=0$ push the motor down, whereas the relative increase in concentration at $\vartheta \approx 2 \pi / 3$ opposes this motion. When full $\mathrm{HI}$ are taken into account, an additional mobility 
factor appears in the integral. The velocity of the fluid relative to the motor (and hence the local mobility) is lower in the stagnant zone near the concave segment and higher near the convex segments. (Mathematically, these tendencies are caused by the term $L_{0}^{\prime} \zeta_{1}$ in (C9).) Thus, the contribution of the concave segment to the integral is reduced even though the magnitude of the concentration distortion (and hence osmotic pressure) is high there, whereas the contribution from the convex surfaces is enhanced. These effects lead to a reversal of the direction of motion. In essence only the convex surfaces are active: the motor is pushed down only at the top $(\vartheta=0)$ and is pushed up at $\vartheta \approx 2 \pi / 3$; as the latter has the greater surface area, it thus wins.

In the limit of small $D a$ the velocity is given by

$$
V_{2}(D a \ll 1)=\alpha_{n} \alpha_{n+1} \Delta^{2} D a \frac{9 n(n-1)(n-2)}{4(2 n+1)(2 n+3)} .
$$

Thus, the velocity of self-propulsion is positive (similar to that found in $\$ 3.2 .1$ ) for $n>2$, whereas for $n=2$ the main contribution is quadratic with respect to $D a$ :

$$
V_{2}(D a \ll 1)=-\alpha_{n} \alpha_{n+1} \frac{81}{280} \Delta^{2} D a^{2} \quad(n=2) .
$$

In the opposite limit, $D a \gg 1$ (fast chemical reaction), one obtains:

$$
V_{2}(D a \gg 1)=-\frac{\alpha_{n} \alpha_{n+1}}{D a} \Delta^{2} \frac{27 n\left(n^{2}-1\right)}{2(2 n+1)(2 n+3)} .
$$

In this case the motor always moves in the direction opposite to that found in the absence of HI (although more slowly by the factor $\Delta^{2}$ ).

Finally, for fanciful motor shapes with $n \gg 1$, the velocity of self-propulsion has the following form:

$$
V_{2}(n \gg 1)=\alpha_{n} \alpha_{n+1} \Delta^{2} \frac{9 D a}{8(1+D a)(2+D a)} n .
$$

Again, the velocity increases linearly as $n$ grows, and the direction of self-propulsion is the same as for large $\Delta$. For large $n$ the expression in brackets in (3.29) vanishes at $D a_{*} \approx(\sqrt{13}-1) n / 6 \approx 0.4343 n$; therefore, the inversion of the motor velocity can be seen only for very fast chemical reactions. The minimum value of $V_{2}$ reached at large $D a \approx 1.300 n$ saturates at the value $-0.8785 \alpha_{n} \alpha_{n+1} \Delta^{2}$ as $n$ grows, in contrast to the maximum of $V_{2}$, which grows as $n$ in this limit.

The calculation of the cubic contribution to the self-propulsion velocity for $\Delta \ll 1$ is too cumbersome even for the simplest case, $\zeta_{1}=\alpha_{3} P_{3}(\mu)$; therefore, this analysis has not been carried out.

\section{Finite surface distortion}

\subsection{No $H I, \Delta \gg 1$}

For numerical computations we deal with the following two-parameter representation of the motor shape:

$$
r_{0}=1+\sum_{n=0}^{4} \alpha_{n} P_{n}(\mu)
$$




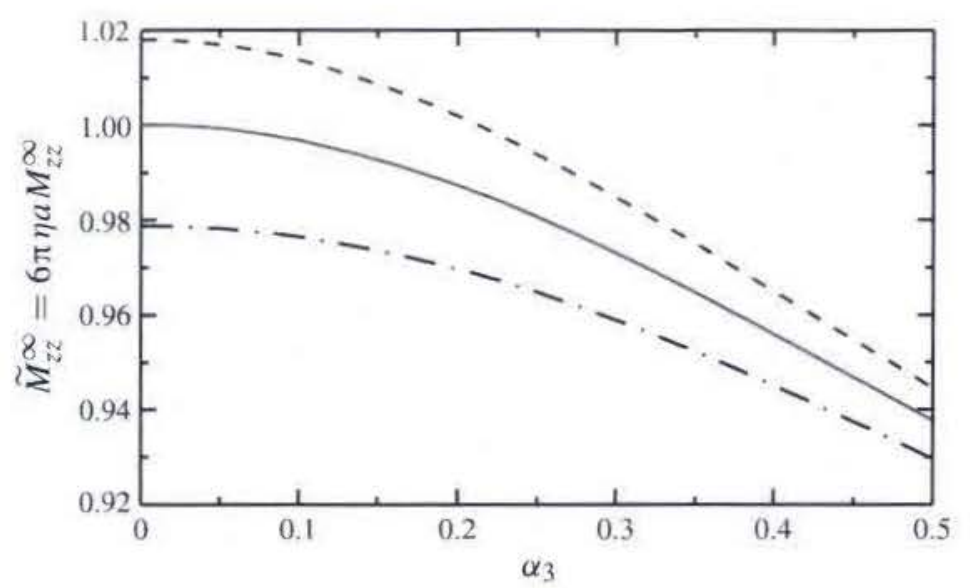

FIGURE 5. (Colour online) The renormalized mobility of the body $\tilde{M}_{z z}^{\infty},(\mathrm{C} 17)$, as a function of $\alpha_{3}$; dot-dashed, solid and dashed lines correspond to $\alpha_{2}=-0.1,0$ and 0.1 , respectively. The particle shape is given by (4.1).

Here $\alpha_{2}$ and $\alpha_{3}$ serve as the parameters, whereas $\alpha_{0}$ and $\alpha_{1}$ are again slaved; they are determined by the conditions:

$$
\begin{aligned}
\int_{-1}^{1} r_{0}^{3} \mathrm{~d} \mu & =2, \\
\int_{-1}^{1} r_{0}^{4} \mu \mathrm{d} \mu & =0,
\end{aligned}
$$

which guarantee that both the volume of the motor and its centre are kept fixed. The conditions (4.2) and (4.3) do not guarantee a unique solution. In computations we follow the branch that is a continuation of $(3,3)$; no bifurcation takes place for this branch within the entire range of $\alpha_{2}$ and $\alpha_{3}$ studied. As stated above, the second condition is not necessary. Moreover, a finite $\alpha_{1}$ also contributes to the shape deviation, not just to the motor displacement. Nevertheless, condition (4.3) is still applied to make the comparison of numerical and analytical results easier. Recall that a change of sign of $\alpha_{3}$ leads only to the reflection of the particle in the $x-y$ plane; therefore $\alpha_{3}$ is set positive below.

The only hydrodynamic characteristic needed to compute the self-propulsion at large $\Delta$ is the constant $\tilde{M}_{z z}^{\infty}$, which is calculated by the boundary element method (Katsikadelis 2002) via the relation (C 17) (see the details in appendix C). An example of the calculations is presented in figure 5, where the variation of $\tilde{M}_{z z}^{\infty}$ with both the coefficients $\alpha_{2,3}$ is shown. It is clear that the change in the mobility does not exceed $10 \%$ - increasing $\alpha_{3}$ decreases $\tilde{M}_{z z}^{\infty}$; in contrast, the mobility grows with increasing $\alpha_{2}$, in agreement with (3.15).

In order to compute the velocity of self-propulsion without $\mathrm{HI}$ one needs the microstructure $g$. The boundary-value problem $(2.7)-(2.9)$ is solved numerically by the boundary element method (Katsikadelis 2002). Results of the computations are shown in figures 6 and 7 . In agreement with the small deformation theory of $\$ 3.2 .2$, for a given volume a motor with a concave segment moves faster; see figures $2(b)$ and $7(a)$ as examples. Again, this effect has a purely geometric origin: the number 


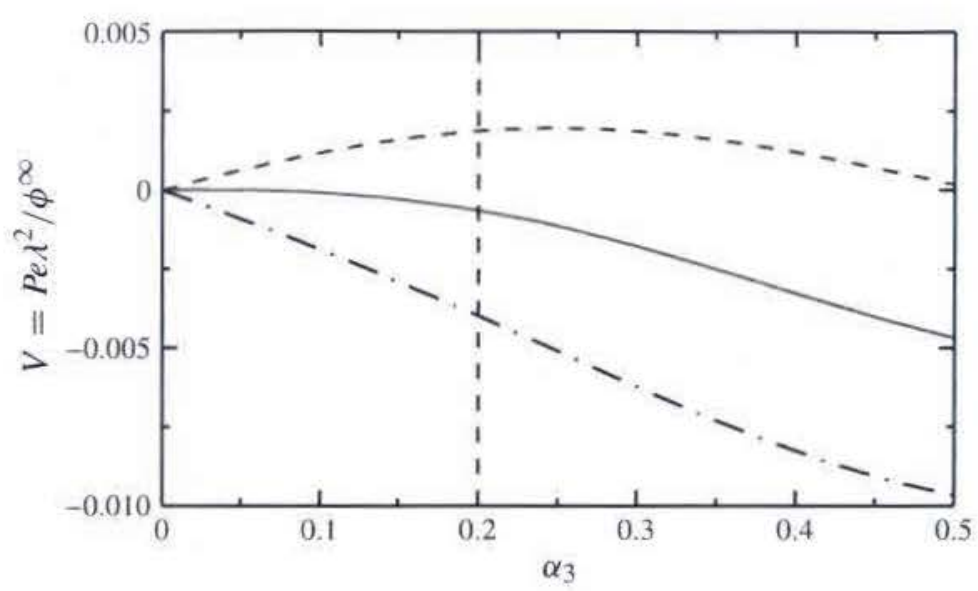

FIGURE 6. (Colour online) Variation of the self-propulsion velocity $V$ with $\alpha_{3}$ for $D a=1$ and $\Delta \gg 1$ (no HI). Dot-dashed, solid and dashed lines are for $\alpha_{2}=-0.1,0$ and 0.1 , respectively. For $\alpha_{3}=0.2$, marked by the vertical line, the concentration fields and motor shapes are depicted in figure 7.

(a)

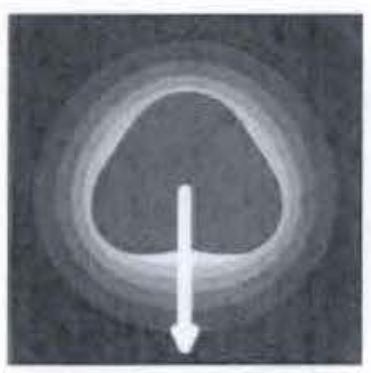

(b)

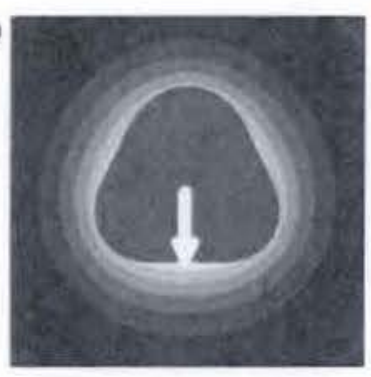

(c)

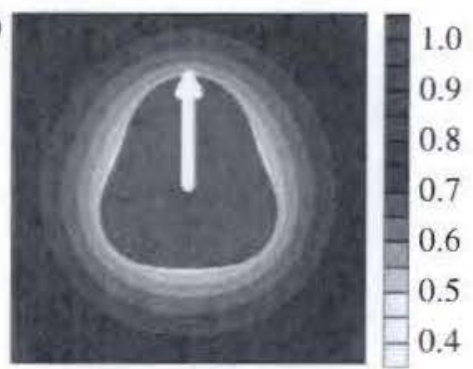

FIGURE 7. (Colour online) Concentration fields for $D a=1$ and motor shapes for $\alpha_{3}=0.2$ and $\alpha_{2}=-0.1(a), 0(b)$ and $0.1(c)$. Arrows show the direction of self-propulsion in the absence of $\mathrm{HI}$, see figure 6 ; for full $\mathrm{HI}$ the direction of motion is opposite to that shown here (see figure 8 ).

of reactant particles that are close to $S_{c}$ and able to react is larger for a unit volume adjacent to a concave segment. Hence, the chemical reaction is more intense at such surfaces, which, in turn, creates a reduction in concentration (i.e. in osmotic pressure) as compared to the unit volume situated near a part of the surface with a positive curvature. This imbalance in the osmotic pressure leads to the overall motion of the motor. It is also seen from figure 7 that there are two local minima in the concentration, one situated at $\pi / 6<\vartheta<\pi / 3$, and the other one at $\vartheta \approx \pi$. These two depletion zones result in opposing forces for self-propulsion; for positive $\alpha_{2}$ (figure $7 c$ ) the former wins, whereas for the negative $\alpha_{2}$ (figure $7 a$ ) the latter one dominates.

The analytical formula for near-spherical particles (3.25) gives good agreement with the numerical results for $\left|\alpha_{2}\right| \leqslant 0.01$ and $\alpha_{3} \leqslant 0$.1. For larger values of $\alpha_{2}$ (e.g. $\alpha_{2}=$ \pm 0.1 shown in figure 6 ), the quadratic term prevails, but only qualitatively agrees with (3.16). The cubic correction proportional to $\alpha_{2}^{2} \alpha_{3}$ should also be included in these cases to achieve quantitative agreement. 


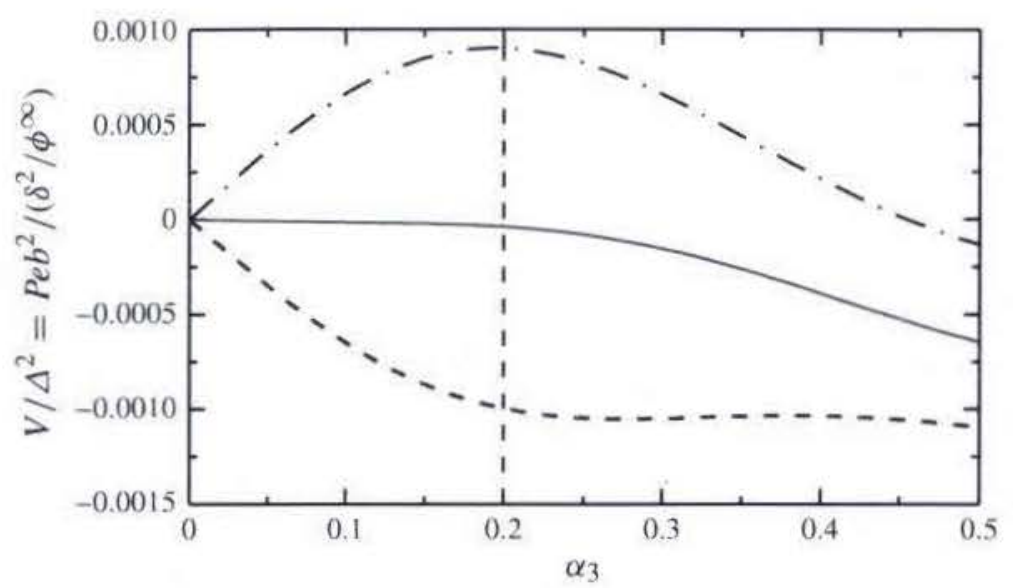

FIGURE 8. (Colour online) Variation of the self-propulsion velocity $V / \Delta^{2}$ with $\alpha_{3}$ for $D a=1$ and $\Delta \ll 1$ (full $\mathrm{HI}$ ); dot-dashed, solid and dashed lines correspond to $\alpha_{2}=-0.1$, 0 and 0.1 , respectively. For $\alpha_{3}=0.2$ marked by the vertical line, the concentration fields and motor shapes are shown in figure 7.

\subsection{Full $\mathrm{HI}, \Delta \ll 1$}

In order to include $\mathrm{HI}$ in the analysis the local mobility tensor is needed; the microstructure $g$ remains the same as discussed in $\$ 4.1$; see, for example, figure 7 . The details of the mobility calculations are discussed in appendix C. It is clear that for full $\mathrm{HI}(\Delta \ll 1) \tilde{M}_{z n} \sim \Delta^{2}$ at $S_{c}$; therefore, within this subsection $V / \Delta^{2}$ is shown.

Results of numerical calculations for small $\Delta$ are shown in figure 8 . As was the case for small departures from a sphere in $\$ 3.3$, HI qualitatively change the phenomenon - the direction of self-propulsion is reversed (see figures 6 and 8). It should be noted that the solid lines corresponding to $\alpha_{2}=0$ are qualitatively the same in figures 6 and 8 , corresponding to both limits $\Delta \ll 1$ and $\Delta \gg 1$. Therefore, for the cubic corrections in small non-sphericity no $\mathrm{HI}$-induced sign reversal takes place and further growth of $\alpha_{3}$ leads to a diminution in the difference between figures 6 and 8 . For both small and large $\Delta$, at rather high $\alpha_{3}$ the motor moves opposite to the $z$ direction independently of the sign of $\alpha_{2}$. For $\alpha_{2}=0.1$ with $\mathrm{HI}$ (the dashed line in figure 8) $V$ remains almost constant for $\alpha_{3}>0.2$, while, in contrast, without $\mathrm{HI}$ $(\Delta \gg 1$ in figure 6$)$ the velocity of self-propulsion continues to grow.

Figure 9 shows the dependence of $V / \Delta^{2}$ on the coefficient $\alpha_{2}$ for rather large $\alpha_{3}=0.4$. For comparison, the results for no $\mathrm{HI}(\Delta \gg 1)$ are also shown. Again, the velocities of self-propulsion with and without $\mathrm{HI}$ are of opposite sign almost everywhere. As seen in the figure, a decrease in $\alpha_{2}$ (for $\alpha_{2}<0$ ) deforms the motor into a heart- or sail-like shape and the velocity of self-propulsion continues to increase (in magnitude) regardless of $\Delta$. In contrast, for large positive $\alpha_{2}$ the velocity saturates. This suggests that a thin sail-like shell will maximize the motor speed for a given volume.

Another notable aspect of the results for $\Delta \ll 1$ is that the absolute values of the velocity of self-propulsion are substantially smaller than for large $\Delta$. (Recall that in comparing the solid and dot-dashed lines in figure 9, one has to keep in mind that $V \sim \Delta^{2} \ll 1$ for full HI.) Indeed, the velocity of self-propulsion with full $\mathrm{HI}$ appears as a competition between two effects: the difference in osmotic pressure associated with the concentration distribution, which pushes the motor as discussed in $\$ 4.1$, and 


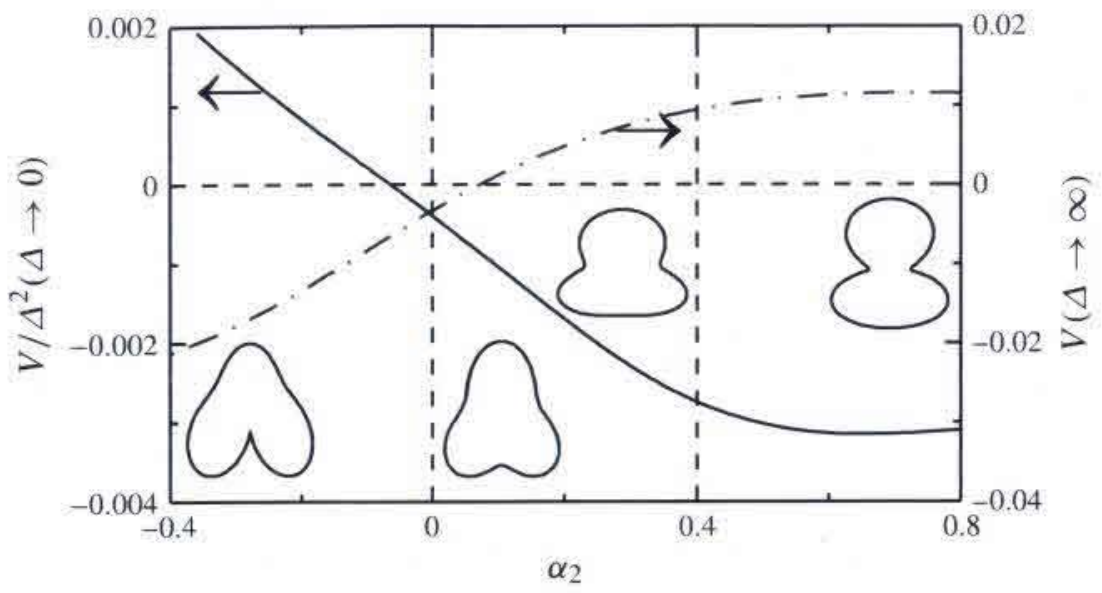

FIGURE 9. (Colour online) Variation of the motor velocity $V / \Delta^{2}$ (left axis, $\Delta \ll 1$, full $\mathrm{HI}$ ) and $V$ (right axis, $\Delta \gg 1$, no $\mathrm{HI}$ ) with $\alpha_{2}$ for $\alpha_{3}=0.4$ and $D a=1$. Motor shapes are shown for $\alpha_{2}=0$ and 0.4 (vertical dashed lines) and $\alpha_{2}=-0.4$ and 0.8 (the vertical solids lines at the two axes). The horizontal dashed line shows $V=0$ for both $\Delta \ll 1$ and $\Delta \gg 1$.

the local hydrodynamics, which reduces the contributions of stagnant zones near the concave elements, leading to the opposite direction of self-propulsion. The smallness of $V / \Delta^{2}$ (at $\left.\Delta \ll 1\right)$ indicates that these two competing tendencies almost compensate each other.

\section{Constant flux motor}

We now discuss the behaviour for another important case of chemical reactioninduced motion: a constant flux of products $j_{s}$ (units of number of particles/(area -time)) prescribed at the motor surface. (The non-uniformly reactive spherical constant flux motor was considered from the colloidal perspective by Córdova-Figueroa et al. 2013). As mentioned in the introduction and $\$ 2$, when product particles are present with diffusivity much smaller than that of the reactant, $D_{P} / D_{R} \ll 1$, and the Damköhler number is small, $D a \ll 1$, the constant flux case can be extracted from the first-order irreversible reaction problem considered in (2.7)-(2.9). In this case, $j_{s}=s \kappa n^{\infty}$, where $s$ is the stoichiometry of the reaction, $R \rightarrow s P$, and, as before, $n^{\infty}$ is the uniform concentration of reactant far from the motor. The self-propulsion now results from the osmotic pressure of the product and the Péclet number is given by

$$
\begin{aligned}
P e_{f} & =\frac{(1+\Delta)^{3}}{\lambda^{2}}\left(\frac{4 \pi}{3} b^{3} \frac{j_{s} r_{c}}{D_{P}}\right) V_{f} . \\
V_{f} & =-\left.\frac{1}{D a} \frac{3}{2} \int_{-1}^{1} \tilde{M}_{z n} g\left(r_{0}, \mu\right)\right|_{D a \ll 1} r_{0} \sqrt{r_{0}^{2}+\left(r_{0}^{\prime}\right)^{2}\left(1-\mu^{2}\right)} \mathrm{d} \mu .
\end{aligned}
$$

Here we define $P e_{f}=U_{f} r_{c} / D_{P}$ via the diffusivity of the product particles, $D_{P}$, and $b$ is now the radius of a product particle. The factor appearing in brackets in $(5.1)$ is the volume fraction of the emitted particles. The Damköhler number $D a=\kappa r_{c} / D_{R}$ is based on the reactant diffusivity and is small. Therefore, $V_{f}$ is independent of the Damköhler number, since $g-1 \sim D a$ for small $D a$. For very rapid reactant diffusion, the reactant 
concentration is hardly distorted from equilibrium and therefore the contribution of the reactant to self-propulsion is $O(D a)$ and is small. In contrast, the product concentration distribution is far out of equilibrium and drives the motor motion. The minus sign in (5.2) indicates the change in the direction of self-propulsion for this type of motor: the motor produces product particles, and where the reaction is most intense, the concentration of products (and the osmotic pressure) is highest.

As before, the actual motor velocity has the form expected for self-diffusiophoresis; from $(5,1)$ and $(5.2)$

$$
U_{f}=\frac{D_{P}}{r_{c}}\left(\frac{4 \pi}{3} r_{c}^{2} b \frac{r_{c}}{a} \frac{j_{s} r_{c}}{D_{P}}\right) V_{f}=\frac{2}{9} \frac{k_{B} T}{\eta}\left(\frac{j_{s}}{D_{P}}\right)\left(\frac{r_{c}^{3}}{a} V_{f}\right),
$$

where now $\left(j_{s} / D_{P}\right)$, with units of number/length ${ }^{4}$, plays the role of the concentration gradient $\nabla n^{\infty}$. Finally, noting that since $j_{s}=s \kappa n^{\infty}, U_{f}$ can be written in a form analogous to $(2,16):\left(j_{s} / D_{P}\right)=s\left(n^{\infty} / r_{c}\right)(D a)\left(D_{R} / D_{P}\right)$ and $D a \rightarrow 0$ while $D_{R} / D_{P} \rightarrow \infty$ such that their product is $O(1)$, and thus $\left(j_{s} / D_{P}\right) \sim\left(n^{\infty} / r_{c}\right)$ and (5.3) has the same form as (2,16). However, the numerical values of $V$ and $V_{f}$, and the direction of motion, differ in the two cases.

\subsection{Small non-sphericity}

For small non-sphericity the results of $\S 3$ are straightforward to extend because, as we stated above, $V_{f}=-\mathrm{d} V / \mathrm{d} D a$ at $D a=0$. The motor shape is again given by (3.1) and (3.2) with $\epsilon$ characterizing the non-sphericity, and the velocity of self-propulsion proceeds as $V_{f}=\epsilon^{2} V_{f 2}+\cdots$. In the absence of HI the quadratic effect is given by

$$
V_{f 2}=-\alpha_{n} \alpha_{n+1} \frac{3 n(n-1)(n+4)}{2(2 n+1)(2 n+3)} .
$$

Cubic effects are readily obtained in the particular case $n=2$ and $\left|\alpha_{2}\right| \ll\left|\alpha_{3}\right| \ll 1$; (3.2.5) for the fixed flux motor becomes

$$
V_{f}=-\epsilon^{2} \frac{18}{35} \alpha_{2} \alpha_{3}+\epsilon^{3} \frac{18}{55} \alpha_{3}^{3} .
$$

The cubic and quadratic effects amplify each other for $\alpha_{2}<0$, corresponding to the particle shape shown in figure 2(b); for positive $\alpha_{2}$ these effects are competing. These results are similar to the reactant-consuming motor; only the overall direction of selfpropulsion changes.

Equation (3.30) can be used for HI, giving the following expression for the velocity of self-propulsion:

$$
V_{f 2}=-\alpha_{n} \alpha_{n+1} \Delta^{2} \frac{9 n(n-1)(n-2)}{4(2 n+1)(2 n+3)} .
$$

For the simplest case of deformation, $n=2$, the velocity vanishes at second order, and thus higher terms, such as $\alpha_{3}^{3}$ and $\alpha_{2}^{2} \alpha_{3}$ need to be included; however, this is beyond the scope of the present paper. It will be shown below in $\$ 5.2$ that this behaviour makes the inclusion of $\mathrm{HI}$ only a quantitative effect; in contrast to the reactant-consuming motor, there is not a large difference in the behaviour for $\Delta \ll 1$ and $\Delta \gg 1$ for the constant flux motor (see figure 12). 
(a)

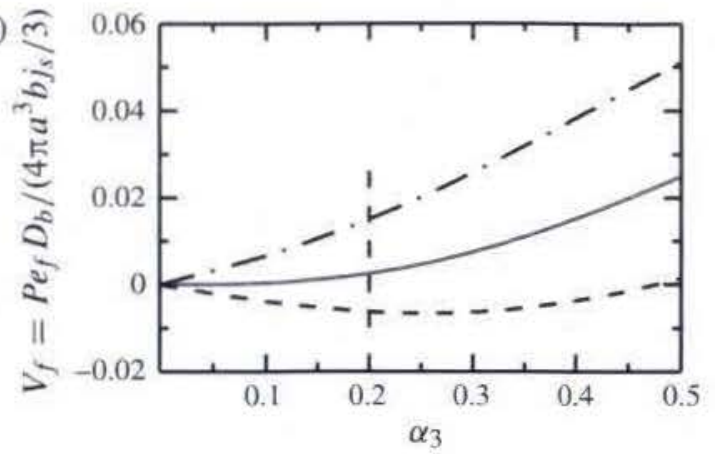

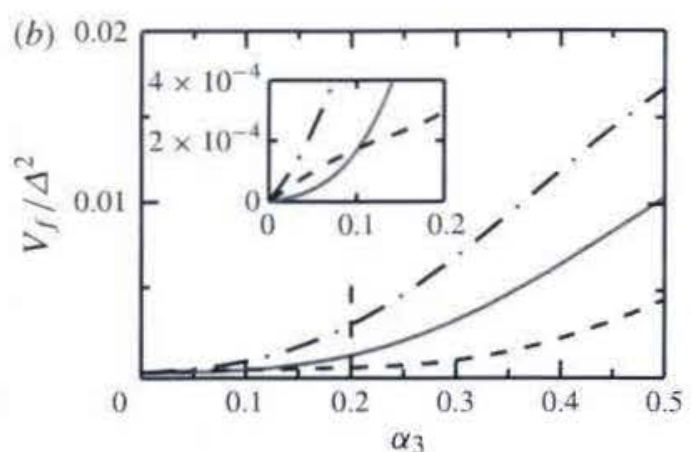

FIGURE 10. (Colour online) Variation of the self-propulsion velocity $V_{f}$, given by $(5.1)$, with $\alpha_{3}$ for the constant flux of product particles for no HI, $\Delta \gg 1(a)$ and full $\mathrm{HI}$, $\Delta \ll 1(b)$. In the latter case $V_{f} / \Delta^{2}$ is shown; the inset presents a zoomed in fragment of panel (b) at small $\alpha_{3}$. The dot-dashed, solid and dashed lines correspond to $\alpha_{2}=-0.1$, 0 and 0.1 , respectively. The shape of the motor is determined by (4.1). For $\alpha_{3}=0.2$ shown by the vertical line, the motor shapes and concentration fields are depicted in figure 11 .

(a)

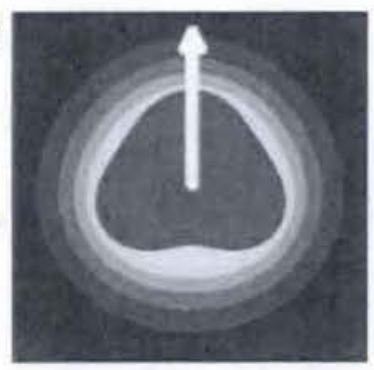

(b)

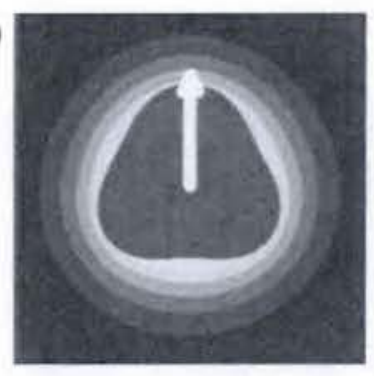

(c)

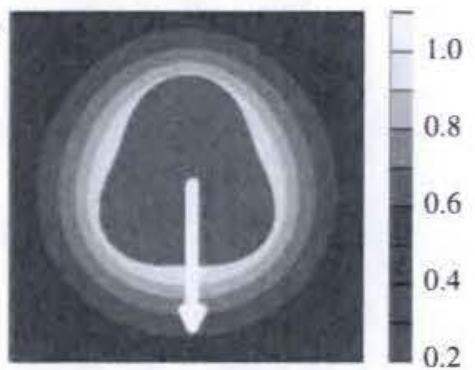

FIGURE 11. (Colour online) Constant flux motor concentration fields and motor shapes for $\alpha_{3}=0.2$ and $\alpha_{2}=-0.1,0$ and $0.1,(a),(b)$ and $(c)$, respectively. The arrows show the direction of self-propulsion for $\Delta \gg 1$ (no $\mathrm{HI}$ ); for $\Delta \ll 1$ (full $\mathrm{HI}$ ) the motor always moves upward. Note that the color scale bar is inverted from that in figure 7 with yellow now representing high product concentration, etc. There is no dependence on the Damköhler for a constant flux motor.

\subsection{Finite non-sphericity}

For finite non-sphericity numerical results are obtained by the boundary element method for the particle shapes represented by (4.1).

The results for no $\mathrm{HI}(\Delta \gg 1)$ are shown in figure $10(a)$, where it is seen that the direction of self-propulsion is opposite to that shown in figure 6 for a reactantconsuming motor because, as pointed out above, regions of low (convex) and high (concave) reactivity decrease (increase) the product concentration perturbations and hence the osmotic pressure. The concentration fields are similarly inverted, as seen by comparing figures 7 and 11. Even with this difference in mind, qualitatively the results for these two problems are quite similar. However, a quantitative difference is noticeable - the numerical values of $V_{f}$ are approximately four times larger than $V$ for the same surface deformation; compare figures 6 and 10. It also worth noting that the approximate solution for small $\alpha_{2,3},(5.5)$, agrees well with the numerical results for $\left|\alpha_{2}\right| \leqslant 0.01$ and $\alpha_{3} \leqslant 0.1$. 


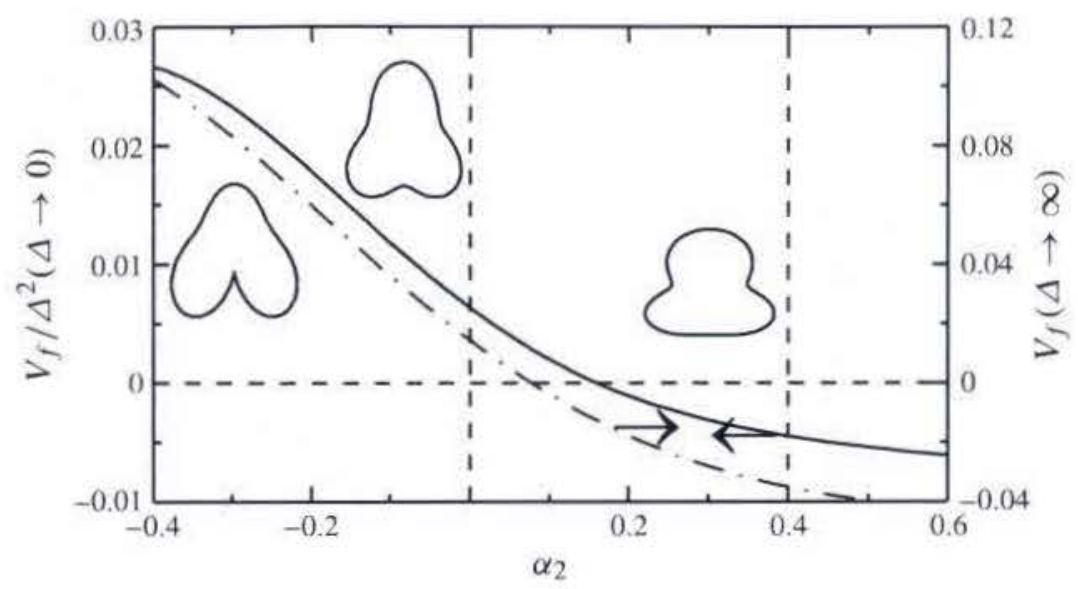

FIGURE 12. Variation of the self-propulsion velocity $V_{f}$ with $\alpha_{2}$ at $\alpha_{3}=0.4$ for the fixed flux of product particles. Left axis: $V_{f} / \Delta^{2}$ for $\Delta \ll 1$ (full HI); right axis: $V_{f}$ for $\Delta \gg 1$ (no HI). The examples of motor shape are presented for $\alpha_{2}=-0.4,0$, and 0.4 . The horizontal dashed line shows $V_{f}=0$ for both $\Delta \ll 1$ and $\Delta \gg 1$.

For full $\mathrm{HI}$, the variation of $V_{f} / \Delta^{2}$ with $\alpha_{3}$ is shown in figure 10(b). In contrast to the motor consuming the reactant, for a constant flux motor the inclusion of HI does not change the situation significantly. In both cases, $\Delta \ll 1$ and $\Delta \gg 1$, the velocity of self-propulsion grows monotonically with a decrease in $\alpha_{2}$. The only exception takes place at small $\alpha_{3}$ and $\alpha_{2} \geqslant 0$, when, as shown in the inset in figure $10(b)$, for full HI $V_{f}$ decreases as $\alpha_{2}$ diminishes, whereas in the opposite limit, $\Delta \gg 1, V_{f}$ becomes negative. This is because the leading term of the asymptotics of weak distortion, $V_{f} \propto$ $\alpha_{2} \alpha_{3}$, vanishes for full HI, and the velocity is governed by the higher nonlinear terms, such as $\alpha_{2}^{2} \alpha_{3}, \alpha_{3}^{3}$ and $\alpha_{2}^{3} \alpha_{3}$. Only the last term, which is rather small in comparison with the others, changes its sign under the sign reversal of $\alpha_{2}$; this is why the lines for $\alpha_{2}= \pm 0.1$ are qualitatively similar in the inset in figure $10(b)$.

The variation of the velocity of self-propulsion with $\alpha_{2}$ shown in figure 12 indicates qualitatively similar trends both with $\mathrm{HI}(\Delta \gg 1)$ and without $\mathrm{HI}(\Delta \ll 1)$. The velocity of self-propulsion grows with decreasing $\alpha_{2}$, when the motor becomes heart-shaped. Again, this suggests that a sail-like motor for a fixed volume maximizes the velocity of self-propulsion.

The qualitative differences between the two types of chemical reaction - a first-order irreversible reaction (figure 9) and constant flux (figure 12) - can be explained as follows. In the absence of $\mathrm{HI}(\Delta \gg 1)$ the behaviour is the same in both cases, only the direction of motion is different. In both cases hydrodynamics reduce the contributions from concave surface elements because the local mobility (the local fluid velocity relative to the motor surface) is small and thus regardless of whether the concentration of reactants is low or that of the products is high the effect of these regions is reduced. For the first-order reaction the local depletion in these zones effectively decreases the rate of consumption, whereas for the second case the flux of particles is fixed independently of the local concentration. Therefore, for the fixed flux motor the pure osmotic effect is more pronounced (this also can be seen comparing the absolute values of $V$ and $V_{f}$ at $\Delta \gg 1$ ), whereas the contribution from hydrodynamics is the same for both types of motors. As we noted above, the osmotic and hydrodynamic effects are well balanced and therefore even a small change in one mechanism is able to reverse the direction of motion for the reactive motor. It 
is also worth noting that the same explanation is valid for the first-order reaction at small $\mathrm{Da}$ (which, of course, is intimately related to the constant flux motor), where again the motor moves in the same direction at small and large $\Delta$.

It is instructive to estimate a typical velocity of self-propulsion for a prototypical motor. From current experiments (Howse et al. 2007) typical parameters are $a=1 \mu \mathrm{m}, \delta=1 \mathrm{~nm}, b=0.1 \mathrm{~nm}$ (so that the limits $b<\delta \ll a$ are satisfied), and $j_{s}=10^{10} \mu \mathrm{m}^{-2} \mathrm{~s}^{-1}$, which provides a velocity of self-propulsion of order $1 \mu \mathrm{m} \mathrm{s}^{-1}$ for an asymmetric reactive Janus particle. The same scaling is also valid for the non-spherical motor, but in this case the velocity should be multiplied by $V_{f} / \Delta^{2}$, which from figure 12 , is smaller by a factor of roughly $10^{-2}$. This suggests that symmetry breaking by reactivity is more effective than by shape.

\section{Conclusions}

In this paper we have considered the motion of a non-spherical osmotic motor a particle of axisymmetric shape moving through a suspension of chemically active particles - which uses chemical energy to sustain its translational motion. The motor is uniformly coated with a catalyst that triggers a first-order chemical reaction on its surface. Owing to the curvature variation along the motor surface, the concentration of the reactant (and, hence, the osmotic pressure) is non-uniform. This non-uniformity, coupled to interparticle interactions between the motor surface and the reactants, results in self-propulsion.

To describe such self-propulsion the colloidal model of Brady (2011) was shown to apply to non-spherical motor and reactant particles. The colloidal treatment allows for any motor shape and for any type of motor-reactant interparticle interaction. For simplicity in the analysis, a hard-sphere potential was considered. It was also shown in appendix $A$ that the same results can be derived from the continuum-level description (in the thin-interfacial limit) of self-diffusiophoresis. Furthermore, the diffusiophoretic model was generalized for arbitrary short-range interactions between the solute and the motor and for a first-order chemical reaction at the motor surface. It was shown that the dimensionless rate of the chemical reaction or Damköhler number $D a$ must be replaced by $\hat{D a}=D a \exp (-\hat{\Phi}(0))$ (cf. (A 10)) in the continuum description.

The problem in the absence of $\mathrm{HI}$ was addressed first for both small and finite distortions of the particle shape from a sphere. For small non-sphericity the result obtained by Wei \& Jan (2010) was shown to be incorrect, as the velocity of selfpropulsion is proportional to at least the squared non-sphericity; no linear effect as found in their paper is present. For a finite-sized distortion numerical computations show that the most efficient design is a motor with a concave surface segment (see figure $2 b$ ), as this leads to the maximum velocity for a given motor volume. It was also shown that the velocity of self-propulsion vanishes in the two limiting cases of small and large values of the Damköhler number. For slow reaction the concentration varies little near the motor and remains almost spherically symmetric, whereas for fast reaction the reactant particles are depleted near the motor surface and there is no osmotic propulsion. The maximum velocity is thus attained for $D a$ close to unity.

When HI between the motor and the reactant were included the behaviour changed dramatically. At small non-sphericity even the direction of self-propulsion is different (except for small Damköhler numbers). This inversion originates from the emergence of stagnant fluid zones near the concave segments of the motor surface that reduce the contribution of these regions to the motor velocity. The concave surfaces provide the largest area for reaction and thus the largest reduction in reactant concentration, which 
then promotes motion of the motor towards the concave surfaces. The local decrease in the hydrodynamic mobility on these portions negates the concentration reduction and reverses the motor's motion.

A rigorous optimization of the motor shape in order to maximize the velocity of self-propulsion was not performed. However, the numerical computations allow us to conclude that the optimal motor should have a concave sail-like shape. Moreover, figure 9 shows that no saturation takes place - a thinner body of large total area produces a larger velocity. These effects hold true for both no HI and full HI, although the direction of self-propulsion is different. Indeed, the 'optimum' motor shape appears to be a thin - to maximize the surface area for reaction - highly curved - to isolate one side of the surface from the reactants - shape, much like a sail; hence the term 'chemical sailing'.

We also analysed the case where a fixed flux of product particles is generated at the motor surface rather than a first-order chemical reaction. The velocity of self-propulsion is opposite in this case, as the concentration (and hence the osmotic pressure) is now highest adjacent to the motor surface. The main qualitative difference is that the inclusion of $\mathrm{HI}$ does not lead to such a drastic change in the motor behaviour as for the motor with the first-order reaction on its surface. In general, both product and reactant particles contribute to the osmotic pressure and the overall velocity of self-propulsion is determined by both the ratio of the diffusivities of the species and the stoichiometry of the chemical reaction. This generalization can be readily performed on the basis of the analysis by Córdova-Figueroa \& Brady (2008); the velocity of self-propulsion needs only to be rescaled by the stoichiometry/diffusivity factor $\left(1-s D_{R} / D_{P}\right)$.

In our analysis we assumed that the motor was large compared to the size of the solute particles, $\lambda=b / a \ll 1$. This limit allows us to neglect the diffusivity of the motor, which is obviously different for diffusion along and perpendicular to the axis of revolution, and it simplifies the inclusion of HI. Nevertheless, this assumption is only technical and can be relaxed, although it leads to a more involved computational problem. Recent work on the related problem of microrheology in which $\lambda$ is varied (from small to large) shows that the no-HI limit applies down to $\Delta$ has small as 1 (Hoh 2013), which may have important implications for the reaction-induced motion of large biological molecules.

Finally, the colloidal expression for the motor velocity (2.3) applies quite generally and leads to various types of motor motion that can be described in an appealing figurative manner. Asymmetric reactivity, such as the Janus motors studied by Golestanian et al. (2005), Golestanian et al. (2007), Córdova-Figueroa \& Brady (2008), Brady (2011) and Córdova-Figueroa et al. (2013), behave in a manner analogous to many swimming microorganisms and this motion is thus called chemical swimming. Propulsion due to an asymmetry in shape, rather than reactivity, as studied here, we call chemical sailing. Brady (2011) noted that the motion of colloidal particles at a fluid-fluid interface due to variation in surface tension caused by the particle releasing a chemical surfactant, which is commonly described as arising from Marangoni flows (see, for example, Ismagilov et al. 2002; Lauga \& Davis 2012; Zhang et al. 2013), can be understood as a manifestation of the same physics that promotes self-diffusiophoresis in bulk, and we refer to this motion as chemical surfing. Motion of a reactive particle can also take place not due to a variation in reactivity or to an asymmetric motor shape, but rather due to a variation in the local hydrodynamics, $\boldsymbol{M}$, or solute-motor interparticle potential, $\hat{\boldsymbol{\Phi}}$, in (2.3) (see also Golestanian et al. 2007). For example, a portion of the motor surface can 
be chemically modified so that the fluid slips along this portion and sticks on the remainder of the motor (Swan \& Khair 2008). Such an asymmetry coupled with a uniform reactivity to drive the solute out of equilibrium can give rise to chemical squirming, in analogy to the motion of squirming microorganisms. Further, Wei \& Jan (2010) showed that two motors can attract each other, leading to behaviour resembling the swarming of biological organisms, a collective motion we call chemical swarming. Thus, chemically driven autonomous motion can display chemical swimming, sailing, surfing, squirming and swarming.

Also, in general, all these different mechanisms and motions can operate simultaneously, which is both advantageous and disadvantageous. The advantage is that the experimentalist has many different options for designing osmotic motors, and thus may be able to tailor motors for specific purposes. The disadvantage is that several mechanisms may compete with one another - for instance, non-uniform reactivity and interactive potential - and cancel out their individual effects, much as $\mathrm{HI}$ reduced the speed of the non-spherical reactive motor studied here. Fortunately, the general formula (2.3) applies to all cases and can thus be used to guide the design of any catalytic nanomotor.

\section{Acknowledgements}

This work was partially supported by the National Science Foundation (Grant No. 1002410 and CAREER Grant No. 1055284 to U.M.C.-F.). This publication is based on work supported by Award No. RUP1-7078-PE-12 (joint grant with the Ural Branch of the Russian Academy of Sciences) of the US Civilian Research \& Development Foundation (CRDF Global) and by the National Science Foundation under Cooperative Agreement No. OISE-9531011.

\section{Appendix A. Diffusiophoretic perspective}

\section{A.1. General potential}

It is instructive to reproduce results analogous to those obtained from (2.6)-(2.9) and (2.12) from a diffusiophoretic point of view. This derivation extends the analysis of Brady (2011) for reactive particles, showing how the conventional diffusiophoretic equations change in the presence of a surface chemical reaction. In this appendix any type of the potential between the solute and the motor, $\hat{\Phi}$, is considered; reduction to a hard-sphere potential is discussed in $\$$ A.2.

It is well-known from Derjaguin et al. (1947) (see also the review by Anderson 1989) that a concentration gradient of solute adjacent to a boundary may cause the fluid to move relative to the surface. The reason for such motion is the presence of a short-range interactive force between a solute molecule and the boundary. A concentration gradient of solute parallel to the surface results in a net local force on the solute whose motion entrains fluid next to the surface. In contrast to conventional diffusiophoresis, where the concentration gradient is externally imposed, a reactive autonomous particle creates the concentration gradient itself, hence the name self-diffusiophoresis.

We consider self-diffusiophoresis in the so-called thin interfacial limit, where the interactive force between the solute and the surface of the motor is short-ranged with length scale $\delta=\Delta a, \Delta \ll 1$, where $a$ is the characteristic size of the motor. The potential of this interactive force normalized by $k_{B} T$ is denoted $\hat{\Phi}(\xi)$ and vanishes when $\xi=O(1)$, where $\xi$ is the local normal coordinate to the surface scaled by $\delta$; the interaction is non-zero only within a layer of thickness $\delta$ near the motor surface. 
The solution proceeds by matched asymptotic expansions with the concentration in the outer region, $n^{(o)}$, satisfying Laplace's equation

$$
\nabla^{2} n^{(o)}=0
$$

while the concentration in the inner region adjacent to the motor surface, $n^{(i)}$, satisfies

$$
\frac{\partial j}{\partial \xi}+\Delta K j=0, \quad j=-\frac{\partial n^{(i)}}{\partial \xi}-n^{(i)} \frac{\partial \hat{\Phi}}{\partial \xi},
$$

where $K=\boldsymbol{\nabla} \cdot \boldsymbol{n}$ is the local (mean) curvature of the motor surface and the $\xi$-derivatives dominant being $O\left(\Delta^{-1}\right)$. The boundary conditions are:

$$
\begin{aligned}
n^{(o)} & =1 \quad \text { as } r \rightarrow \infty, \\
j & =-\Delta \operatorname{Dan}^{(i)} \quad \text { at } \xi=0, \\
n^{(o)} & =n^{(i)} \quad \text { as } r \rightarrow r_{0}, \quad \xi \rightarrow \infty,
\end{aligned}
$$

representing the fixed concentration far from the motor, the mass balance condition in the presence of the chemical reaction at the motor surface and the matching condition, respectively. (The matching condition includes the continuity of all needed derivatives.) It is worth noting that $\xi=0$ corresponds to the surface of the solid core, $S_{h}$, so that it is parameterized by $r=r_{0}^{(-)}(\mu)$. The boundary-value problem (A 1)-(A 5) is non-dimensionalized with $n^{\infty}$ and $a$ (or $r_{c}=a+\delta$, since the difference between $a$ and $r_{c}$ results only in $O(\Delta)$ corrections) used for the scales for the concentration and length, respectively. The Damköhler number $D a=\kappa r_{c} / D_{b}$, with $D_{b}$ the diffusivity of the reactants (which are taken to be so small in size $b \ll \delta$ such that there are no HI between a reactant particle and the motor surface - in the continuum diffusiophoretic perspective the reactant has no size).

The solution of the inner problem (A 2), (A 4), (A 5) proceeds as a power series in $\Delta: n^{(i)}=n_{0}^{(i)}+\Delta n_{1}^{(i)}+\cdots$. At zeroth order the flux $j_{0}$ vanishes according to (A 4); therefore $n_{0}^{(i)}$ is given by the Boltzmann distribution

$$
n_{0}^{(i)}=n^{(o)}\left(r=r_{0}\right) \mathrm{e}^{-\hat{\phi}},
$$

which provides the matching with the yet-unknown outer solution $n^{(o)}(\boldsymbol{r})$. This result coincides precisely with the known behaviour in the absence of chemical reaction, $D a=0$ (Derjaguin et al. 1947; Anderson 1989), and agrees with the recent results by Sharifi-Mood, Koplik \& Maldarelli (2013) for small Da. The surface chemical reaction will only change the outer solution; the solute distribution across the interaction layer is just the Boltzmann distribution (provided $\Delta D a \ll 1$; if $D a \rightarrow \infty$, the concentration would drop to zero in the thin layer near the surface and remove the driving force for fluid motion).

To first order the mass flux $j_{1}$ is constant and therefore the solution for $n_{1}^{(i)}$ has the following form:

$$
n_{1}^{(i)}=C_{1} \mathrm{e}^{-\hat{\phi}}-j_{1} \mathrm{e}^{-\hat{\phi}} \int_{0}^{\xi} \mathrm{e}^{\hat{\phi}} \mathrm{d} \xi,
$$

where the constant $j_{1}$ follows from the boundary condition (A 4):

$$
j_{1}=-\operatorname{Dan}^{(o)}\left(r=r_{0}\right) \mathrm{e}^{-\hat{\phi}(0)} .
$$


Matching of the flux between the inner and outer regions, $\boldsymbol{n} \cdot \boldsymbol{j}^{(o)}=\boldsymbol{n} \cdot \boldsymbol{j}^{(i)}$, as $\xi \rightarrow \infty$ and $r \rightarrow r_{0}$, gives the boundary condition

$$
\boldsymbol{n} \cdot \nabla n^{(o)}=\hat{D} a n^{(o)} \quad \text { at } r=r_{0},
$$

where

$$
\hat{D a}=D a \exp (-\hat{\Phi}(0)),
$$

which makes the boundary-value problem (A 1), (A 3), and (A 9) complete. The outer boundary value problem is as expected, but with a Damköhler number containing the reactant-motor interparticle potential $\hat{\Phi}$. Since the reaction occurs at the actual motor surface, the local concentration of reactant there differs from that just outside the inner region, $n^{(o)}\left(r \rightarrow r_{0}\right)$, by the Boltzmann factor $\mathrm{e}^{-\hat{\Phi}(0)}$. It is also seen that this problem coincides with (2.7)-(2.9) for the microstructure from the colloidal perspective. (Recall that we have taken the potential to be hard-particle-like at the distance $\delta$ and the chemical reaction to occur at this same surface, thus $\hat{\Phi}(0)=0$.)

While (A 1), (A 3) and (A 9) were derived for uniform reactivity on the motor surface, it is easy to see that they apply equally well for non-uniform reactivity: the only change needed is to note that $\kappa$ in the definition of $D a$ now varies along the surface. (In particular, the same rescaling of $D a$ can be extracted from the recent derivation in Sharifi-Mood et al. (2013), although the authors deal with small Da only.) These equations can also be applied to the constant flux problem, which was shown in $\S 5$ to be obtained from the $D a \rightarrow 0$ limit, and thus the results from the diffusiophoretic model of Golestanian et al. (2005, 2007) apply.

The diffusiophoretic slip velocity $\boldsymbol{u}^{\text {slip }}$ as $\xi \rightarrow \infty$ and the velocity of self-propulsion $U$ can now be obtained from the standard expressions (Anderson 1989):

$$
\begin{aligned}
\boldsymbol{u}^{\text {slip }} & =\frac{k_{B} T \lambda_{D}^{2}}{\eta} \nabla_{\|}\left[n\left(r=r_{0}\right)\right], \\
U & =-M_{z z}^{\infty} \oint_{S_{i}} \boldsymbol{u}^{\text {slip }} \cdot \boldsymbol{\sigma} \cdot \boldsymbol{n} \mathrm{d} S,
\end{aligned}
$$

respectively, where $\lambda_{D}^{2}=\int_{0}^{\infty} \xi(1-\exp (-\hat{\Phi})) \mathrm{d} \xi$, with $\lambda_{D}$ being the Derjaguin length, and the gradient operator is along the motor surface. To obtain the second formula, the reciprocal theorem for Stokes flow is applied with one pair (velocity, stress tensor) chosen as $\left(\boldsymbol{U} \boldsymbol{e}_{z}+\boldsymbol{u}^{\text {slip }}, \boldsymbol{\Sigma}\right)$ and the second pair $(\boldsymbol{v}, \boldsymbol{\sigma})$ corresponding to the Stokes flow past the (non-spherical) motor.

\section{A.2. Hard-sphere potential}

We now discuss the model simplifications for the particular case of a hard-sphere potential considered in the text. As noted above, the rescaling of the Damköhler number according to $(\mathrm{A} 10)$ is not needed because the chemical reaction has been already shifted to the contact surface $S_{c}$, where $\hat{\Phi}=0$.

The Derjaguin length can be easily calculated, $\lambda_{D}^{2}=\delta^{2} / 2$, which gives the following expression for the velocity of self-propulsion

$$
U=-\frac{k_{B} T \delta^{2}}{2 \eta} M_{z z}^{\infty} \oint_{S_{c}} \nabla_{\|}\left[n\left(r=r_{0}\right)\right] \cdot \boldsymbol{\sigma} \cdot \boldsymbol{n} \mathrm{d} S .
$$


This value has to be compared with $(2,6)$ from the colloidal perspective. It is clear that $\boldsymbol{U}$ given by (2.6) can be rewritten as

$$
\boldsymbol{U}=U \boldsymbol{e}_{z}, \quad \text { with } U=-n^{\infty} k_{B} T M_{z z}^{\infty} \oint_{S_{\tau}} g \boldsymbol{v} \cdot \boldsymbol{n} \mathrm{d} S,
$$

where the relation $M_{z n}=M_{z z}^{\infty} \boldsymbol{v} \cdot \boldsymbol{n}$ has been used, which is discussed in appendix C.

This value of $U$ can be easily converted to (A 13). Indeed, noting that $\boldsymbol{\nabla} \cdot \boldsymbol{v}=0$, as it is a solution to Stokes equations,

$$
\oint_{S_{c}} g \boldsymbol{v} \cdot \boldsymbol{n} \mathrm{d} S=\int_{\delta V} \boldsymbol{v} \cdot \nabla g \mathrm{~d} V=\delta \oint_{S_{c}} \mathrm{~d} S \int_{0}^{1} \mathrm{~d} \xi \boldsymbol{v} \cdot \nabla \boldsymbol{g},
$$

where $\delta V$ is the volume between two surfaces, $S_{c}$ and $S_{h}$, and $\xi$ again is the normal coordinate to both the surfaces scaled by $\delta$. ( $S_{c}$ obviously corresponds to $\xi=1$.) Near the solid surface $v \approx \eta^{-1} \sigma_{n \tau} \delta \xi \boldsymbol{\tau}+O\left(\delta^{2}\right)$, where $\boldsymbol{\tau}$ is the tangential vector to the solid surface, which permits calculating the integral over $\xi$. The final expression for $U$ reads

$$
U=-\frac{n^{\infty} k_{B} T \delta^{2}}{2 \eta} M_{z z}^{\infty} \oint_{S_{c}}\left(\nabla_{\tau} g\right) \sigma_{n \tau} \mathrm{d} S,
$$

which agrees with (A 13). Therefore, all the results presented in $\S \S 3-5$ for $\Delta \ll$ $1(\delta \ll a)$ can be reproduced from the continuum point of view.

\section{Appendix B. Solutions for $g_{1}$ and $g_{2}$ at small non-sphericity}

It is clear that both Laplace's equation (2.7) and the condition at the infinity (2.9) remain the same at all orders of expansion in $\epsilon$. Thus, below, only the boundary condition (2.8), which is shifted to the spherical surface $r=1$, is given.

At first order one obtains

$$
\begin{aligned}
g_{1 r}-D a g_{1} & =\left(-g_{0 r r}+D a g_{0 r}\right) \zeta_{1}, \\
g_{1} & =\sum_{n=2}^{\infty} \frac{\alpha_{n} A_{n}}{r^{n+1}} P_{n}(\mu), \quad A_{n}=\frac{2+D a}{n+1+D a} A .
\end{aligned}
$$

(Recall that $A=-D a /(1+D a)$ comes from $g_{0}$, see (3.13).) Within this section the subscripts are used to denote the corresponding partial derivatives. This solution coincides with that given in Wei \& Jan (2010), except for the terms proportional to $P_{0}(\mu)$ and $P_{1}(\mu)$, which should not be present as these correspond to a volume change $\left(P_{0}(\mu)\right)$ or a shift in the particle centre $\left(P_{1}(\mu)\right)$.

In fact, if we add to the expressions (3.2) and (B 2) the terms corresponding to $n=1$, the velocity of self-propulsion from $(2,12)$ requires the concentration at the curved surface of the motor, which is given by

$$
g\left(r=1+\epsilon \zeta_{1}, \mu\right) \approx g_{0}(r=1, \mu)+\epsilon\left(g_{1}+\frac{\partial g_{0}}{\partial r} \zeta_{1}\right)_{r=1}+O\left(\epsilon^{2}\right) .
$$

The contribution proportional to $\epsilon$, which could result in self-propulsion, vanishes:

$$
\left(g_{1}+\frac{\partial g_{0}}{\partial r} \zeta_{1}\right)_{r=1}=A_{1} \alpha_{1} \mu-A \alpha_{1} \mu=0
$$


because $A_{1}=A$ according to (B 2). Thus $g$ is constant at the motor surface with the accuracy of $\epsilon^{2}$; the uniform contribution clearly does not lead to the self-propulsion. Wei \& Jan (2010) in their derivation omitted the second term of this contribution and therefore mistakenly concluded that propulsion would occur at $O(\epsilon)$.

At second order the boundary condition reads:

$$
\begin{aligned}
g_{2 r}-\operatorname{Dag}_{2}= & D a\left(\frac{1}{2} g_{0 r r} \zeta_{1}^{2}+g_{0 r} \zeta_{2}+g_{1 r} \zeta_{1}\right)-\left(\frac{1}{2} g_{0 r r} \zeta_{1}^{2}+g_{0 r r} \zeta_{2}+n_{1 r r} \zeta_{1}\right) \\
& +\left(1-\mu^{2}\right)\left(g_{1 \mu} \zeta_{1 \mu}+\frac{1}{2} g_{0 r} \zeta_{1 \mu}^{2}\right) .
\end{aligned}
$$

There is no need to solve the entire second-order problem, only the part proportional to $\mu$ is necessary to calculate $V_{2}$. This part has the following form

$$
g_{2}^{(1)}=\frac{A_{1} \mu}{r^{2}},
$$

with

$$
A_{1}=\sum_{n=2}^{\infty} \frac{C_{n}(n+2) \alpha_{n} \alpha_{n+1}}{2+D a}\left[\frac{2 n+3}{n+2} D a A_{n}+(3+D a) A_{n+1}-\left(\frac{12+5 D a}{n+2}-n\right) A\right] .
$$

\section{Appendix C. Calculation of the hydrodynamic mobility}

As stated in $\S 2$, calculation of the mobility $\boldsymbol{M}$ (up to the sign) is reduced to finding the Stokes flow $\boldsymbol{u}$ around the particle under a unit force. From a computational point of view, it is simpler to calculate the Stokes velocity $v$ around the motionless motor when a velocity $\boldsymbol{e}_{z}$ is imposed far from it. It is obvious that $\boldsymbol{u}=-\boldsymbol{M}_{z z}^{\infty} \boldsymbol{v}$ and, hence, the needed component of the mobility is given by

$$
M_{z n}=M_{z z}^{\infty} v_{n}
$$

Of course, a similar relation is valid for the normalized mobility, which differs by the factor $6 \pi$ from the unscaled analogue:

$$
\tilde{M}_{z n}=\tilde{M}_{z z}^{\infty} v_{n} .
$$

In order to calculate the velocity field for a slightly deformed sphere, we introduce the vector potential $\psi \boldsymbol{e}_{\phi}$, according to the relation:

$$
\boldsymbol{v}=\boldsymbol{\nabla} \times\left(\psi \boldsymbol{e}_{\phi}\right),
$$

where $e_{\phi}$ is the unit vector in the azimuthal direction.

The amplitude $\psi$ of the vector potential satisfies the equation

$$
D^{2} \psi=0, \quad D=\nabla^{2}-\frac{1}{r^{2} \sin ^{2} \vartheta}
$$

with the boundary conditions

$$
\psi=\nabla_{n} \psi=0 \quad \text { at } r=r_{0}^{(-)} \text {. }
$$


The velocity components are expressed via $\psi$ as:

$$
v_{r}=\frac{1}{r \sin \vartheta} \frac{\partial(\sin \vartheta \psi)}{\partial \vartheta}, \quad v_{\vartheta}=-\frac{1}{r} \frac{\partial(r \psi)}{\partial r},
$$

so that $\psi$ is related to the conventional streamfunction $\psi_{c}$ (Happel \& Brenner 1965) by the relation:

$$
\psi_{c}=r \sin \vartheta \psi
$$

For small non-sphericity, when $r_{0}$ is given by (2.10), the boundary conditions (C 5) are prescribed at $r_{0}^{(-)}=a_{-}+\epsilon \zeta_{1}+O\left(\epsilon^{2}\right)$, where $a_{-}=a / r_{c}=1 /(1+\Delta) \approx 1-\Delta+\Delta^{2}$ is the mean radius of the solid core.

At first order the solution for a slightly non-spherical particle was found by Sampson (1891) (see also Happel \& Brenner 1965). However, in the current paper a different parametrization of the surface (in terms of the Legendre polynomials rather than the Gegenbauer polynomials) is used, so it is useful to present the solution at the needed order of approximation.

The solution to (C 4) and (C 5) is given by

$$
\begin{aligned}
\psi= & \frac{a_{-}}{4}\left(2 \frac{r}{a_{-}}+\frac{a_{-}^{2}}{r^{2}}-3\right) P_{11}(\mu)-\epsilon \frac{3}{4}\left(\frac{a_{-}}{r}-\frac{r}{a_{-}}\right) \\
& \times\left(\frac{a_{-}}{r}\right)^{n}\left\{\frac{\alpha_{n+1} P_{n 1}}{2 n+3}-\frac{\alpha_{n} P_{n+1,1}}{2 n+1} \frac{a_{-}}{r}\right\}+\text { o.t. }
\end{aligned}
$$

Here $P_{n 1}$ are the associated Legendre polynomials (Abramowitz \& Stegun 1965) and o.t. denotes the omitted terms proportional to $\alpha_{n} P_{n-1,1}$ and $\alpha_{n+1} P_{n+2,1}$, unimportant for the further analysis. Equation (C 8) agrees with the classical result by Sampson (1891). Moreover, using the result by Payne \& Pell (1960), one can readily extract (3.15) from (C 8).

Completing this part of the work, we represent the final expression for $\tilde{M}_{z n}$ at $r=r_{0}$ with the needed accuracy:

$$
\tilde{M}_{z n}=-L_{0} \mu-\epsilon\left(L_{0}^{\prime} \mu \zeta_{1}-M_{0}\left(1-\mu^{2}\right) \zeta_{1}^{\prime}-L_{n} P_{n+1}+L_{n+1} P_{n}\right)
$$

where

$$
\begin{aligned}
L_{0}=1-\frac{3}{2} a_{-}+\frac{1}{2} a_{-}^{3}, & L_{0}^{\prime}=\frac{3}{2} a_{-}\left(1-a_{-}^{2}\right), \quad M_{0}=1-\frac{3}{4} a_{-}-\frac{1}{4} a_{-}^{3}, \\
L_{n}= & \frac{3}{4} \frac{\alpha_{n}}{2 n+1}(n+1)(n+2) a_{-}^{n}\left(a_{-}^{2}-1\right), \\
L_{n+1} & =\frac{3}{4} \frac{\alpha_{n+1}}{2 n+3} n(n+1) a_{-}^{n-1}\left(a_{-}^{2}-1\right) .
\end{aligned}
$$

Here $L_{0}$ and $M_{0}$ are the conventional mobility functions (calculated at $r=1$ ) for a sphere of radius $a_{-}$, which represents the radial and meridional components of the Stokes flow velocity; $L_{0}^{\prime}$ is the $r$-derivative of $L(r)$ at $r=1$. The final two terms, $L_{n}$ and $L_{n+1}$, stem from the $O(\epsilon)$ terms in the Stokes flow (C 8). 
In the limit of small $\Delta$, these coefficients reduce to:

$$
\begin{aligned}
L_{0} & \left.=\frac{3}{2} \Delta^{2}, \quad L_{0}^{\prime}=3 \Delta\left(1-\frac{3}{2} \Delta\right), \quad M_{0}=\frac{3}{2} \Delta\left(1-\frac{1}{2} \Delta\right), \quad \text { (C. } 13 a-c\right) \\
L_{n} & =\alpha_{n} \frac{n+1}{2 n+1} \hat{L}_{n}, \quad \hat{L}_{n}=-\frac{3}{2}(n+2) \Delta\left(1-\frac{2 n+1}{2} \Delta\right),
\end{aligned}
$$

Collecting all the terms, one arrives at (3.26).

In the opposite limiting case $(\Delta \gg 1)$, of course $L_{0}=M_{0}=1$, whereas the other terms are zero. This allows a comparison of the results with those presented in $\$ 3.2,1$.

For finite non-sphericity the problem is solved by the boundary element method, based on the well-known integral representation of the velocity at the solid surface:

$$
\boldsymbol{v}(\boldsymbol{r})-\boldsymbol{v}^{\infty}(\boldsymbol{r})=\frac{1}{8 \pi} \oint_{S_{c}} \boldsymbol{J}\left(\boldsymbol{r}-\boldsymbol{r}^{\prime}\right) \cdot \boldsymbol{\sigma}\left(\boldsymbol{r}^{\prime}\right) \cdot \boldsymbol{n} \mathrm{d} S\left(\boldsymbol{r}^{\prime}\right),
$$

where $\boldsymbol{J}$ is the Stokeslet (Green's dyadic) and $\sigma$ is yet-to-be-determined stress tensor. At the particle surface $v=0$, and therefore this equation is a linear integral equation for $\boldsymbol{\sigma}$. Numerical calculation of $\boldsymbol{\sigma} \cdot \boldsymbol{n}$ allows the velocity at any point $\boldsymbol{r}$ to be found. With the stress tensor known one can readily find the mobility of the particle:

$$
M_{z z}^{\infty}=\left(\boldsymbol{e}_{z} \cdot \oint_{S_{c}} \boldsymbol{\sigma} \cdot \boldsymbol{n}\right)^{-1},
$$

and calculate $M_{z n}$ via (C 1).

Technically, it is even simpler to calculate the velocity of self-propulsion via (A 16) using the continuum approach. (The computation of the normal component of the velocity near the solid wall results in large computational errors.) Note that this result already contains the factor $\Delta^{2}$ and therefore the integral can be calculated over either $S_{c}$ or $S_{h}$, with the difference between them proportional to $\Delta^{3}$.

\section{REFERENCES}

ABRamowitz, M. \& STEgun, I. A. 1965 Handbook of Mathematical Functions: with Formulas, Graphs, and Mathematical Tables. Dover Publications.

ANDERSON, J. L. 1989 Colloidal transport by interfacial forces. Annu. Rev. Fluid Mech. 21, 61-99.

BRADY, J. F. 2011 Particle motion driven by solute gradients with application to autonomous motion: continuum and colloidal perspectives. J. Fluid Mech. 667, 216-259.

Córdova-FigueroA, U. M. \& BRADY, J. F. 2008 Osmotic propulsion: the osmotic motor. Phys. Rev. Lett. 100, 158303.

Cordova-Figueroa, U. M., Brady, J. F. \& Shklyaev, S. 2013 Osmotic propulsion of colloidal particles via constant surface flux. Soft Matt. 9, 6347-6542.

Derjaguin, B. V., Sidorenkov, G. P., Zubashchenkov, E. A. \& Kiseleva, E. V. 1947 Kinetic phenomena in boundary films of liquids. Kolloidn. Z 9, 335-347.

Golestanian, R., Liverpool, T. B. \& AJdARI, A. 2005 Propulsion of a molecular machine by asymmetric distribution of reaction products. Phys. Rev. Lett. 94, 220801.

Golestanian, R., LIVERPool, T. B. \& AJDARi, A. 2007 Designing phoretic micro- and nanoswimmers. New J. Phys. 9, 126. 
Happel, J. \& Brenner, H. 1965 Low Reynolds Number Hydrodynamics. Prentice-Hall.

$\mathrm{HOH}$, N. J. 2013 Effects of particle size ratio on single particle motion in colloidal dispersions. $\mathrm{PhD}$ thesis, California Institute of Technology.

Howse, J. R., Jones, R. A. L., Ryan, A. J., Gough, T., Vafabakhsh, R. \& Golestanian, R. 2007 Self-motile colloidal particles: from directed propulsion to random walk. Phys. Rev. Lett. 99, 048102.

Ismagilov, R, F., Schwartz, A., Bowden, N. \& Whitesides, G. M. 2002 Autonomous movement and self-assembly. Angew. Chem. Int Ed. Engl. 41, 652-654.

Katsikadelis, J. T. 2002 Boundary Elements Theory and Applications. Elsevier.

LaUGA, E. \& Davis, A. M. J. 2012 Viscous Marangoni propulsion. J. Fluid Mech. 705, 120-133.

Paxton, W. F., Sundararajan, S., Mallouk, T. E. \& Sen, A. 2006 Chemical locomotion. Angew. Chem. Inil Ed. Engl. 45, 5420-5429.

PAYNe, L. E. \& Pell, W. H. 1960 The Stokes flow problem for a class of axially symmetric bodies. J. Fluid Mech. 7, 529-549.

Popescu, M. N., TAsinkeVyCh, M. \& Dietrich, S. 2011 Pulling and pushing a cargo with a catalytically active carrier. Eur. Phys. Lett. 95, 28004.

RÚckner, G. \& Kapral, R. 2007 Chemically powered nanodimers. Phys. Rev. Lett. 98, 150603.

Russel, W. B., Saville, D. A.\& Schowalter, W. R. 1989 Colloidal Dispersions. Cambridge University Press.

Sampson, R. A. 1891 On Stokes's current function. Phil. Trans. R. Soc. 182, 449-518.

Sengupta, S., Ibele, M. E. \& Sen, A. 2012 Fantastic voyage: designing self-powered nanorobots. Angew. Chem. Intl Ed. Engl. 51, 8434-8445.

Sharifi-Mood, N., Koplik, J. \& Maldarelli, C. 2013 Diffusiophoretic self-propulsion of colloids driven by a surface reaction: the sub-micron particle regime for exponential and van der Waals interactions. Phys. Fluids 25, 012001.

SWAN, J. W. \& KhaIR, A. S. 2008 On the hydrodynamics of 'slip-stick' spheres. J. Fluid Mech. 606, $115-132$.

Thakura, S. \& Kapral, R. 2010 Self-propelled nanodimer bound state pairs. J. Chem. Phys. 135, 204505 ,

WEI, H. -H. \& JAN, J. -S. 2010 Self-propulsion and dispersion of reactive colloids due to entropic anisotropy. J. Fluid Mech. 657, 64-88.

Zhang, H., DuAn, W., LiU, L. \& SEN, A. 2013 Depolymerization-powered autonomous motors using biocompatible fuel. J. Am. Chem. Soc. 135, 15734-15737. 